\title{
State of the Art in the Aeroelasticity of Wind Turbine Blades: Aeroelastic Modelling
}

\author{
Lin Wang ${ }^{\text {a, }}$, Xiongwei Liu ${ }^{\mathrm{b}}$, Athanasios Kolios ${ }^{\mathrm{a}}$ \\ ${ }^{a}$ Centre for Offshore Renewable Energy Engineering, School of Water, Energy and Environment \\ Cranfield University, Cranfield, Bedford, MK43 0AL,UK \\ ${ }^{\mathrm{b}}$ Entrust, The Innovation Centre, Sci-Tech Daresbury, Daresbury, Cheshire, WA4 4FS, UK
}

\begin{abstract}
With the continuous increasing size and flexibility of large wind turbine blades, aeroelasticity has been becoming a significant subject for wind turbine blade design. There have been some examples of commercially developed wind turbines experiencing aeroelastic instability problems in the last decade, which spokes for the necessity of aeroelastic modelling of wind turbine blades. This paper presents the state-of-the-art aeroelastic modelling of wind turbine blades, provides a comprehensive review on the available models for aerodynamic, structural and cross-sectional analysis, discusses the advantages and disadvantages of these models, and outlines the current implementations in this field. This paper is written for both researchers new to this research field by summarising underlying theory whilst presenting a comprehensive review on the latest studies, and experts in this research field by providing a comprehensive list of relevant references in which the details of modelling approaches can be obtained.
\end{abstract}

Keywords: Wind turbine blade; Aeroelasticity; Aeroelastic modelling; Aerodynamic model; Structural model; Cross-sectional analysis model

\section{Introduction}

The capacity and dimension of commercial wind turbines have being increased exponentially over the last three decades, from a rated power of $75 \mathrm{~kW}$ and a rotor diameter of $17 \mathrm{~m}$ for earlier designs up to a rated power of $7.5 \mathrm{MW}$ and a rotor diameter of over $125 \mathrm{~m}$ for modern machines. The trend of increasing size of large wind turbines is expected to continue in the next decade. The power rating of commercial wind turbines has gone up to $8 \mathrm{MW}$ [1], and the potential for 10-20MW wind turbines is being investigated $[2,3]$.

${ }^{*}$ Corresponding author. Tel.: +44(0)1234754706; E-mail address: lin.wang@cranfield.ac.uk 
The increasing size of large wind turbines lowers the cost of wind power generation in term of levelised cost of energy $(\$ / \mathrm{kWh})$; however it introduces significant aeroelastic effects, which are caused by the interaction of aerodynamic loads, elastic deflections and inertial dynamics. Specifically, during the operation of a wind turbine, the blades experience elastic deflections due to aerodynamic loads exerted by the airflow passing the blades. The deformed blades affect, in turn, the flow field around the blades, which in return influences the aerodynamic loads on the blades. The inertia dynamics play a significant role in the correlation between the aerodynamic loads and elastic deflections, and the resulting accelerations. The blade can experience oscillation due to the aeroelastic effects, and it becomes unstable under harmonic conditions and/or when the damping is negative.

Aeroelastic effects might result in instability problems, such as edgewise instability and flutter, which can be devastating both to the blades and the wind turbine. For instance, as reported in Ref. [4], $0.5 \%$ of the LM (Lunderskov Mobelfabrik) $19 \mathrm{~m}$ wind turbine blades were damaged within one year. These blades were mounted on $600 \mathrm{~kW}$ wind turbines around the world and were damaged due to blade edgewise instability. The changes in wind turbine blade design due to the growth in size might lead to other not yet recognised aeroelastic instabilities. Therefore, investigating the aeroelasticity characterisation of large wind turbine blades is playing an important role in the development of large wind turbines.

An aeroelastic model should contain an aerodynamic part to calculate the aerodynamic loads and a structural part to determine the structural dynamic responses. In aeroelastic modelling, wind turbine blade structure is often represented as a series of 1D beam elements, which are characterised by the cross-sectional properties of the blade, such as cross-sectional stiffness and mass per unit length. It should be noted that wind turbine blades are generally made of composite materials and have a complicated structural layout. Due to the intrinsic nature of composite materials and the complexity of blade structural layout, obtaining the cross-sectional properties of the composite blades is quite challenging and requires a specialised cross-sectional analysis model. Fig. 1 presents the components of the aeroelastic modelling of wind turbine blades, and each component is reviewed in this paper.

In order to develop more advanced and reliable aeroelastic models for wind turbine blades, it is important to have a view of the state of the art in aeroelastic modelling of the blades. For this concern, a comprehensive review of the aeroelastic modelling of wind turbine blades has been developed within this paper. 
This paper is structured as follows. Sections 2, 3 and 4 review the aerodynamic, structural and cross-sectional analysis models, respectively. Section 5 presents the current implementations, followed by a discussion in Section 6 and a conclusion in Section 7 .

\section{Aerodynamic model}

In order to perform aeroelastic modelling of wind turbine blades, an aerodynamic model should be included to calculate the aerodynamic loads exerted by the airflow passing over the blades. Four types of aerodynamic models have been used in the aeroelastic modelling of wind turbine blades, i.e. BEM (blade element momentum) model, vortex model, actuator type model and CFD (computational fluid dynamics) model.

\subsection{BEM (blade element momentum) model}

The BEM model was originally proposed by Glauert [5] by combining blade element theory and blade momentum theory. The blade element theory discretises the blade into several elements and ignores the mutual influence between two adjacent elements, as shown schematically in Fig. 2 reproduced from Ref. [6]. The aerodynamic loads on each element depend on its local airfoil characteristics, i.e. its lift and drag coefficients. The sum of these loads yields the total loads on the blade. The blade momentum theory introduces axial induction factor $a$ and angular induction factor $a^{\prime}$ to respectively calculate the induced velocity in the axial and tangential directions, as shown schematically in Fig. 3. The induced velocity affects the angle of attack of the blade and therefore influences the aerodynamic loads calculated by the above blade element theory. Combining blade element theory and blade momentum theory provides a solution to obtain the performance parameters of each blade element through an iterative procedure [6, 7].

The original BEM model has several limitations which are usually found in wind turbine applications. The majority of these limitations have been overcome through introducing empirical corrections borrowed from helicopter applications or based on wind turbine experience.

\subsubsection{Tip loss}

One of the main limitations of the original BEM model is that it ignores the effects of vortices shedding from the blade tip on the induced velocity. Practically, these effects play a significant role in the induced velocity distribution along the blade, especially the region near the blade tip. In order to compensate for this deficiency in the BEM model, several tip loss correction factors have been proposed. Prandt1 [8] proposed a tip loss correction factor through modelling the wake of the wind turbine as vortex sheets. Prandtl's tip loss correction is simple and efficient and also 
improves the accuracy in the predictions of induced velocity distribution. Based on the NavierStokes solutions, $\mathrm{Xu}$ and Sankar [9] proposed a correction of the Prandtl model, showing good agreement with the experimental data of the NREL Phase VI rotor [10].

\subsubsection{Turbulent wake state}

Another limitation of the original BEM model is that the model becomes invalid when the axial induction factor $a$ is larger than around 0.4. This occurs for the cases when wind turbines operate at high tip speed ratios, e.g. fixed-speed wind turbines at low wind speeds, as the blade gets into turbulent wake state $(a>0.5)$. For the turbulent wake state, the wind velocity behind the blade, calculated based on blade momentum theory, becomes negative, which is obviously unreasonable. The original BEM model is incapable of providing a reasonable thrust coefficient when the blade operates in the turbulent wake state. In order to overcome this limitation of the BEM model, several empirical models have been proposed, such as Glauert model [11], AeroDyn model [12], Buhl model [13], GH-Bladed model [14], Burton model [15] and Spera model [16]. Fig. 4 (reproduced from Ref. [17]) presents a comparison of these models. From Fig. 4 we can see that all these models agree well with the experimental data except for the Spera model.

\subsubsection{Dynamic inflow}

The induced velocities calculated using the original BEM model are quasi-steady, which implies the wake is in equilibrium with the inflow. Practically, if the inflow is changed, before a new equilibrium is achieved there exists a time delay, which is a function of the rotor diameter and wind speed [18]. Fig. 5 (reproduced from Ref. [18]) depicts the predicted and measured dynamic response on the rotor shaft torque of the Tjaereborg $2 \mathrm{MW}$ wind turbine [19] for a sudden change in pitch angle. At $t=2 \mathrm{~s}$, the pitch angle is changed from $0^{\circ}$ to $3.7^{\circ}$, reducing the local angle of attack. The rotor shaft torque firstly decreases from 260 to $150 \mathrm{kNm}$, and then gradually increases, taking approximately $10 \mathrm{~s}$ delay to reach a new equilibrium state with a value of around $220 \mathrm{kNm}$. At $t=32 \mathrm{~s}$, the pitch angle is adjusted back to $0^{\circ}$, and a similar time delay in the rotor shaft torque response is observed. Taking account of this time delay needs a dynamic inflow model. Several empirical dynamic inflow models have been developed, such as Øye model [20] and PittPeters model [21]. The comparison of these models in Ref. [22] shows that all these models agree well with the trends of measurements. Recently, Henriksen et al. [23] proposed a simplified dynamic inflow model, in which the induced axial wind speed states are gathered in a single averaged induced axial wind speed. The simplified dynamic inflow model has been demonstrated as being able to capture the most significant dynamics of the more advanced dynamic inflow model, and its application in wind turbines can be found in Ref. [24]. 


\subsubsection{Dynamic stall}

Dynamic stall is the nonlinear unsteady aerodynamic effect that occurs when airfoils quickly change the angle of attack, and it is associated with the separation of the boundary layer. During the dynamic stall, the boundary layer initially separates at the trailing edge, and gradually shifts to the leading edge with an increasing angle of attack [25]. The angle of attack of rotating blades changes dynamically due to sudden change in wind, such as wind shear and atmospheric turbulence. The response on aerodynamic loads introduced by changing the angle of attack has a time delay and depends on whether the boundary layer is attached or separated. In the case of an attached boundary layer, the time delay can be calculated using the Theodorsen theory for unsteady lift [26]. In the case of a separated boundary layer, the separation point position needs to be taken into account in the calculation of the time delay. The dynamic stall phenomenon has been evident from the measurement of aerodynamic coefficients on practical wind turbine blades. One example illustrated in Fig. 6 (reproduced from Ref. [27]) is the dynamic stall event measured at the 30\% span position of the CER (Combined Experiment Rotor). As can be seen from Fig. 6, due to the dynamic stall effects, the airfoil normal force coefficient $C_{N}$ changes dynamically with the angle of attack and is significantly different from the value measured in static conditions. A number of dynamic stall models have been developed, such as BL (Beddoes-Leishman) model [28], ONERA model [29], Snel [30] model, Øye [31] model, BV (Boeing-Vertol) model [32] and Risø [33] model. Comparisons of different dynamic stall models can be found in Refs. [34, 35], showing that all these models show reasonable agreement with experimental data but none of the models is significantly better in all cases than the others.

\subsubsection{Other corrections}

Various other corrections have been proposed to correct the BEM model, including 1) stall delay corrections [36-38], which account for stall delay due to the rotation of the blade; 2) radial flow corrections [39], which take account of radial flow in the calculation of aerodynamic loads; and 3) skewed wake corrections [40, 41], which account for skewed wakes introduced by nonaxisymmetric flow (e.g. wind turbines operating at yaw angles relative to the incoming wind). These corrections further improve the accuracy of the BEM model.

\subsubsection{Validity and applications}

The validity of the BEM model has been extensively established by comparing it with experimental data [42-45]. Because it is simple, efficient and well-proven, the BEM model has become a standard method for analysing the aerodynamic performance of wind turbine blades. Additionally, the high efficiency of the BEM model makes it suitable for blade design 
optimisation, which generally involves a large number of case studies. Based on the BEM model and different optimization strategies, authors have conducted a series of case studies on the design optimization of wind turbine blades [7, 46-49].

Compared to other aerodynamic models, the BEM model is fast and able to provide accurate results when reliable airfoil aerodynamic data are available. For this reason, the BEM model has been used for the aerodynamic part by most wind turbine aeroelastic models.

\subsection{Vortex model}

In order to better model the wake dynamics of wind turbine rotors, the vortex model [50], in which the trailing and shed vorticity in the wake are represented by lifting lines or surfaces, has also found applications in the aeroelastic models of wind turbine blades. Fig. 7 (reproduced from Ref. [51]) presents an example of the wake modelling of a two-bladed wind turbine rotor using the vortex model.

The wake in vortex models can be calculated using either the prescribed-wake method or freewake method.

In a prescribed-wake method, the wake shedding from the blade is assumed to be rigid and is described using semi-empirical formulations. The applications of prescribed-wake vortex models in analysing the wakes of wind turbine blades can be found in Refs. [52, 53]. The prescribed-wake in these models saves computation time but limits their application to steady incoming flow.

A free-wake method, in which the wake can be varied freely both in time and space, is necessary for unsteady flow. A typical layout of the free-wake modelling of a wind turbine blade is presented in Fig. 8 (reproduced from Ref. [54]).

Free-wake vortex models have been applied to wind turbine blades to study the unsteady wakes of the blades $[51,55,56]$, and the aeroelasticity of the blades [54, 57]. The free-wake method used in these models enables them to be capable of handling a complex unsteady flow, e.g. dynamic inflow. However, the free-wake method is much more computationally expensive than the prescribed-wake method.

Compared to the BEM model, the vortex model requires more computational resources, and it tends to diverge due to the intrinsic singularities of the vortex panels in the developing wake. Additionally, viscous effects are ignored in the vortex model, which limits its application to wind turbines to some extent. 


\subsection{Actuator type model}

In the actuator type model, the wind turbine blade is represented by a disc/line/surface with distributed loads on the disc/line/surface. Various forms of actuator type model have been developed, which can be classified based on the representation of the blade, namely: actuator disc model, actuator line model and actuator surface model.

The actuator disc model is possibly the earliest model used for studying rotor performance. The classical actuator disc model, which is derived from the 1D momentum theory initially developed by Rankine [58] and Froude [59], is ended up with BEM model [5]. In its general form, however, the actuator disc can also be numerically combined with the Euler or Navier-Stokes equations.

In a numerical actuator disc model, the Euler or Navier-Stokes formulations are typically solved by finite volume or difference scheme, as in a usual CFD calculation. However, the flow around the blades and the geometry of the blades are not resolved. The surface of the blade is replaced by distribution forces acting on the incoming flow.

In the simple case of a uniformly loaded actuator disc, the force acting on the disc is determined by thrust coefficient and reference wind speed. In the case of a non-uniformly loaded actuator disc, the force acting on the disc varies along the radial location but remains constant over the annulus. Similar to BEM, the local forces on the blades can be calculated using the lift and drag coefficients of the airfoil section. A relevant issue is the determination of the local angle of attack to find the lift and drag coefficients. Shen et al. [60] provided a method to determine the local angle of attack according to information slightly upstream of the blade plane.

Sørensen and Shen [61] extended the non-uniformly loaded actuator disc method to the actuator line approach, in which the blade forces are represented using a line with distributed loads. The wake modelling of a three-blade wind turbine rotor based on the actuator line model is illustrated in Fig. 9 (reproduced from Ref. [61]). In their later study [62], the actuator line approach is applied to the aerodynamic modelling of the MEXICO (Model Experiments in Controlled Conditions) rotor, showing good agreement with the measurements.

Shen et al. $[63,64]$ further extended the actuator line approach to the actuator surface method, in which the wind turbine blade was represented by a planar surface. Sibuet Watters and Masson [65] proposed their actuator surface method using a slightly different approach. Kim et al. [66] proposed an improved actuator surface model for wind turbine analyses, overcoming the need for tip loss correction. 
The actuator surface method needs not only the lift and drag coefficient of airfoils, but also the skin friction and pressure distribution on the airfoil surface. Dobrev et al. [67] used a linear function, which is determined from the lift and drag coefficients, to represent the pressure distribution in the actuator surface method.

The actuator type models discussed above should be granted the credit for providing a better insight into the 3D (three-dimensional) flow development and the credit for contributing to a better understanding of wake dynamics. However, solving the Navier-Stokes equations is more time-consuming than BEM, and the actuator type models, in which loads on the blade are still calculated based on blade element theory and tabulated airfoil data, do not predict aerodynamic loads more accurately than the BEM model.

\subsection{CFD (computational fluid dynamics) model}

With the advancement of computing resources, CFD has received considerable attention in recent years. The CFD method solves the governing equations of fluid flow at thousands of positions on and around the blade in an iterative process, which does not require predetermined airfoil aerodynamic data for the calculation. In addition to aerodynamic load calculations, CFD is also a valuable tool to visualise the flow field around the blade, as shown in Fig. 10 from Ref. [68].

To perform CFD modelling of wind turbine blades, the 3D blade geometry needs to be exactly described in a digitised format. Wind turbine blades often have a complex geometric shape with varied spanwise cross-sectional information, i.e. airfoil shape, chord and twist angle distributions. The 3D blade geometry is generally constructed using CAD (computer aided design) software, such as SolidWorks [69] and UG [70].

Due to the complex geometry of a wind turbine blade, it is quite challenging to generate an appropriate mesh for the CFD modelling of the blade. There are three typical types of mesh, i.e. structured, unstructured and hybrid, as illustrated in Fig. 11. Structured mesh has advantages in high resolution, easy convergence and low memory usage. However, it is difficult and timeconsuming to generate a structured mesh for complex geometries, such as highly twisted blades. The major advantage of unstructured mesh is the ease of mesh generation for complex geometries. However, unstructured mesh consumes more computation time, as it generally results in higher cell numbers than structured mesh filling the same volume. Hybrid mesh, also known as adaptive mesh, is the combination of structured and unstructured meshes. In hybrid mesh, structured mesh is used for important regions, such as boundary layers, while unstructured mesh is used elsewhere. Due to the flexibility of hybrid mesh, it has been widely used for the mesh generation of CFD modelling of wind turbine blades [71-73]. 
The mathematical fundamentals of CFD are the NS (Navier-Stokes) equations [74], which are the governing equations of fluids derived from the momentum, energy, and continuity conservations. For wind turbine blades, the relative wind speed is much lower than the speed of sound and therefore the flow can be considered incompressible [75]. The governing equations of incompressible NS equations (convective form) are given by [76]:

$$
\begin{gathered}
\frac{\partial \mathbf{u}}{\partial \mathrm{t}}+(\mathbf{u} \cdot \nabla) \mathbf{u}=-\nabla P+v \nabla^{2} \mathbf{u} \\
\nabla \cdot \mathbf{u}=0
\end{gathered}
$$

where $\mathbf{u}$ is the flow velocity, $P$ is the pressure divided by the density, $v$ is the (constant) coefficient of kinematic viscosity.

The discretisation of NS equations can be achieved through three typical discretisation methods, i.e. FVM (finite-volume method), FEM (finite-element method) and FDM (finite-difference method). FVM is a common method used in CFD modelling, as it has advantages in solution speed and memory usage. FEM is mainly utilised in structural analysis, but it can also be applied to fluids. For instance, ANSYS CFX [77], a widely used commercial CFD software package, is based on FEM. Compared to FVM, FEM is much more stable, but it consumes more memory and has slower solution times [78]. FDM is easy to implement, but it is limited to simple grids. Currently, FDM is only utilised in a few specialised CFD codes.

Directly solving NS equations, known as DNS (direct numerical simulation), requires huge computational resources, which exceed the capacity of current computers. In order to apply NS equations to solve practical engineering problems on wind turbine blades, some kind of turbulence modelling is required. Currently, turbulence models are mainly derived based on RANS (Reynolds Averaged NS equations), which give approximate time-averaged solutions to NS equations. Various RANS-based turbulence models have been used for wind turbine applications, such as the $k-\omega$ SST model [79], $k-\varepsilon$ model [80] and Spalart-Allmaras model [81]. Among these models, the $k-\omega$ SST model is found to be most successful for both 2D airfoil and 3D blade CFD modelling [82-84].

A number of studies have been performed on the CFD modelling of stall-regulated wind turbines, showing that all RANS-based turbulence models fail to accurately model the stalled flow at a high angle of attack [18]. Possible solutions to this problem are to use more complicated turbulence modelling approaches, such as 1) LES (large eddy simulations) [85], which retains large eddies and ignores small eddies in solving NS equations; and 2) DES (detached eddy simulations) [86], which is a hybrid method combining RANS and LES. However, both LES and DES are much more computationally costly than RANS, as they require considerably finer computational meshes and the computations have to be carried out with time-dependent (unsteady) analysis. 
Due to its high fidelity, CFD has been extensively used for wind turbine wake modelling. Bechmann et al. [87] investigated the wake behind MEXICO wind turbine using CFD with RANS turbulence model, showing good agreement with experimental data. AbdelSalam and Ramalingam [88] studied the wake characteristics in the near and far wake regions of wind turbine blades using CFD and compared the results against both experimental data and actuator disc model. The results from the CFD model showed better agreement with the experimental data than the actuator disc model. A review of CFD for wind turbine wake aerodynamics was presented in Ref. [89], in which different CFD techniques for modelling the rotor and the wake were discussed. The general review of wind turbine wake aerodynamics can be found in Refs. [75, 90], and the general review of wind turbine aerodynamics can be obtained from Refs. [91, 92].

Studies have been carried out to combine CFD with simpler aerodynamic models to develop hybrid models. Esfahanian et al. [93] developed a hybrid CFD/BEM model to predict the aerodynamic performance of wind turbine blades. In the hybrid model, CFD was used to calculate 2D aerodynamic coefficients of a series of spanwise sections of the blades, and BEM was used to simulate the 3D flow field through the wind turbine blade. The hybrid model was demonstrated to be much faster than merely CFD while able to retain desirable accuracy. Suzuki et al. [94] developed a hybrid CFD/vortex model to simulate wind turbine aerodynamics at yaw angles by coupling an unsteady CFD model and a prescribed wake model. The results were compared to both experimental data and a free wake model, showing that hybrid CFD/vortex model provided better predictions than the free wake model for low yaw angles. Sturge et al. [95] presented a hybrid technique combining actuator disc and full rotor CFD for wake modelling of wind turbine, showing more rapid and accurate calculations than using either method on its own.

CFD has been applied to the aeroelastic modelling of both wind turbine blades [96-98] and the full turbine [99-101], showing promising results and providing a better understanding of the flow field around the deformed blades and the turbine. Fig. 12 from Ref. [97] presents an example of air speed distribution around the deformed blade obtained from the CFD-based aeroelastic modelling. Currently, CFD is still computationally too expensive, which is the main obstacle of its industrial applications in aeroelastic modelling.

\section{Structural model}

In order to perform aeroelastic modelling of wind turbine blades, a structural model needs to be included to determine the structural dynamic response of the blade. Structural models used in aeroelastic modelling of wind turbine blades can be roughly categorized into two groups, i.e. 3D FEM model and 1D equivalent beam model. In the 3D FEM model, wind turbine composite blades are generally discretised using 3D composite shell elements, which are capable of 
describing composite layer characteristics throughout the shell thickness. For the 1D equivalent beam model, in order to discretise the blade into a series of 1D beam elements, three types of discretisation methods are often used in the aeroelastic modelling of wind turbine blades, i.e. modal approach, MBD (multi-body dynamics) and 1D FEM.

\subsection{D FEM (finite-element method) model and 1D beam model}

Wind turbine blade structures can be modelled using either a 3D FEM model with shell elements or a $1 \mathrm{D}$ beam model with beam elements.

\subsubsection{D FEM model}

In the 3D FEM model, wind turbine composite blades are generally constructed using 3D composite shell elements, which are capable of describing composite layer characteristics throughout the shell thickness. An example of a 3D FEM model of wind turbine composite blades is illustrated in Fig. 13.

Due to the complicated aerodynamic shape and structural layout of a wind turbine composite blade, generating a 3D FEM model of the blade using general-purpose commercial finite-element packages, such as ANSYS [102] and Abaqus [103], is tedious and time-consuming. In order to facilitate the generation of 3D FEM models of wind turbine blades, Berg and Resor developed a specialised tool called NuMAD v2.0 (Numerical Manufacturing And Design version 2.0) [104], which is a stand-alone pre-processor for ANSYS. NuMAD v2.0 provides a user-friendly GUI (graphic user interface), as depicted in Fig. 14, for defining the blade geometry information (such as chord and twist angle distributions) and the blade structural layout information (such as shear web locations and composites layup). The output from NuMAD v2.0 is a series of APDL (ANSYS Parametric Design Language) commands used to generate the 3D FEM model of the blade in ANSYS. In addition to the creation of the finite element model, NuMAD v2.0 includes some advanced capabilities, such as tabularised input format and output for CFD mesh creation.

3D FEM is an incredible tool for examining the detailed stress distributions within a blade, and it has been applied to aeroelastic modelling of wind turbine blades by coupling it with an aerodynamic model. 3D FEM is generally coupled with CFD to perform aeroelastic modelling of wind turbine blades [96-98]. The aeroelastic modelling based on coupled 3D FEM and CFD is able to provide accurate results, but it is computationally expensive. A way to save computational cost is to couple the 3D FEM with an efficient aerodynamic model, e.g. BEM model. Verelst [105] studied the aeroelasticity of wind turbine blades based on an FEM-BEM coupled approach, in which the finite element program Abaqus is coupled with the a BEM code WT_Perf [106]. This 
approach is an attempt to develop an efficient aeroelastic model, but it is still not efficient enough and has not been widely used in industrial applications in the aeroelastic modelling of wind turbine blades.

\subsubsection{D beam model}

Wind turbine blades are slender structures having one of their dimensions significantly larger than the other two. Such structures can be efficiently modelled using beam models. The beam axis is defined along the largest dimension, and a cross section perpendicular to this axis is considered to be varying along the span of the beam. An example of a beam model of wind turbine blades is presented in Fig. 15.

A number of beam models exist and they can be roughly categorised into two groups, i.e. linear and nonlinear.

Two widely used linear beam models are the Euler-Bernoulli beam model [107] and the Timoshenko beam model [108]. The Euler-Bernoulli beam model, also known as the classical beam model, deals with slender beams subjected to extensional, torsional and bending loads. The shear deformation effects are ignored in the model. The Timoshenko beam model was developed by Timoshenko in the early $20^{\text {th }}$ century. This model takes account of shear deformation effects, making it more suitable for describing the behaviour of thick and short beams than the EulerBernoulli beam model. Regarding wind turbine blades, which generally have a thin and slender structure, the Timoshenko beam model shows little difference from the Euler-Bernoulli beam model. Due to its easy implementation, the Euler-Bernoulli beam model has been used by most structural models in the aeroelastic modelling of wind turbine blades.

Both the Euler-Bernoulli and Timoshenko beam models contain the assumption of small deflections. This assumption is invalid for very flexible blade design because such blades often experience large deflections. Handling large deflections requires a nonlinear beam model, taking account of the geometric nonlinearities caused by large deflections. A well-known example is the GEBT (geometrically exact beam theory) [109], in which the deformed beam geometry (i.e. the displacements and rotations of the beam reference line) is represented exactly.

Compared to the 3D FEM, the 1D beam model is much faster, saves much computation time and is capable of providing accurate results if constructed properly. Therefore, almost all aeroelastic models represent the blades as a series of 1D beam elements instead of 3D shell elements. Authors [110] developed a nonlinear aeroelastic model called NAM_WTB (Nonlinear Aeroelastic Model for Wind Turbine Blades) based on the nonlinear beam model GEBT. NAM_WTB takes account 
of large blade deflections and geometric nonlinearities, and thus greatly improves the accuracy of the aeroelastic analysis of wind turbine blades. The results from NAM_WTB show good agreement with experimental data, and its nonlinear capability has been demonstrated through case studies.

\subsection{Discretisation methods of the $1 \mathrm{D}$ beam model}

In order to discretise the blade into a series of 1D beam elements, three types of discretisation methods are often used in the aeroelastic modelling of wind turbine blades, i.e. modal approach, MBD and 1D FEM.

\subsubsection{Modal approach}

In the modal approach, the deflection shape of the flexible bodies, such as the blade and tower, is described as a linear combination of a set of mode shapes, which are usually obtained from a finite element pre-processor. Fig. 16 (reproduced from Ref. [111]) presents an example of first and second mode shapes of a wind turbine blade.

Using mode shapes is an effective way to reduce the number of DOFs (degrees of freedom) and therefore reduce the size of matrices and speed up the computations per time step. Therefore, the modal approach is computationally efficient, resulting in a rapid simulation. For this reason, the majority of the present commercial wind turbine blade aeroelastic models use the modal approach to calculate the structural dynamics of the blades.

However, the flexibility of the modal approach is restricted somewhat by its restraints on the type and number of DOFs allowed in the structure. For instance, FLEX5 [112], which is a commercially widely used aeroelastic analysis model based on the modal approach, uses only the initial three or four (two flapwise and one or two edgewise) eigenmodes for the blade. The torsional eigenmodes, which are important for flutter analysis, are generally not available in modal-approach-based aeroelastic models.

Another major limitation of the modal approach is that it is inherently limited to linear analysis due to its linear assumption, i.e. the deflection shape of the flexible components must be a linear combination of the provided mode shapes. This means that the modal approach is not capable of handling the large deflections of the flexible blade.

Additionally, the accuracy of the modal approach greatly depends on the prescribed mode shapes. In order to obtain the mode shapes of the blade, a finite-element-based pre-processor is required. 


\subsubsection{MBD (multi-body dynamics) method}

In the MBD method, the structure is discretised into a number of bodies, which can be either flexible or rigid. These bodies are interconnected by force elements (such as springs) or kinematic constraints (such as joints) $[113,114]$. The dynamics of the structure can then be evaluated using equations of motion, which are usually derived from Lagrange's equations or Newton-Euler equations.

The MBD method benefits from high modelling flexibility due to its capability to generate and couple together an arbitrary number of separate bodies in a single dynamic system. Fig. 17 (reproduced from Ref. [115]) presents an example of wind turbine configuration based on MBD. From Fig. 17 we can see that the three blades (flexible bodies) are attached to the hub (rigid body) using joints called 'pitch hinges', which allow the relative pitching motion of the blade with respect to the hub. Compared to the modal approach, the MBD method requires more computational resources, but it enables an increased number of DOFs to be modelled.

\subsubsection{D FEM method}

The 1D FEM approach finds approximate solutions of 1D beam problems by the analysis of an assemblage of finite elements, which are interconnected by nodes, as illustrated in Fig. 18. The 1D FEM allows a more comprehensive and accurate deformation description of wind turbine blades, and it only requires slightly more computational resources than the other two discretisation approaches. Therefore, the 1D FEM has been adopted by most of recently developed aeroelastic models of wind turbine blades [18].

\section{Cross-sectional analysis model}

Wind turbine blades generally are made of composite materials due to their high strength-toweight ratio and good fatigue performance. To construct a 1D beam model of wind turbine blades for aeroelastic modelling, the cross-sectional properties of the blade, such as mass per unit length and cross-sectional stiffness, are essential pieces of information. Fig. 19 (reproduced from Ref. [116]) depicts the structural layout of a typical blade cross-section, comprising three cells with two shear webs. As can be seen from Fig. 19, each cell includes several laminates, each of which is made up of several plies, and each ply is a composite mat placed at different angles, resulting in a complicated structural topology. Due to the intrinsic nature of composite materials and the complexity of blade structural topologies, it is quite challenging to obtain the cross-sectional properties of a wind turbine blade. 
In order to obtain the cross-sectional properties of wind turbine blades, various cross-sectional analysis models have been developed, which can be categorised into three groups, i.e. 3D FEMbased model, 2D FEM-based model and CLT (classical lamination theory)-based model.

\subsection{D FEM based model}

The most complex method to calculate the cross-sectional properties of wind turbine blades is based upon 3D FEM. 3D FEM, despite its capability for accurate displacement and stress analysis, cannot directly yield the cross-sectional properties of wind turbine blades. It relies upon computationally complex post-processing of force-displacement data. One such post-processing tool is BPE (Blade Properties Extractor) [117], which has been developed by Sandia National Laboratories and Global Energy Concepts. As illustrated in Fig. 20 (reproduced from Ref. [118]), BPE applies unit loads at the blade tip and then transfers the displacement results of the 3D FEM model of the blade to several MATLAB routines, which calculate the stiffness matrices for the equivalent beam elements. In principle, BPE should be capable of providing the most accurate cross-sectional properties because all 3D information can be captured by the 3D FEM model. However, it seems the BPE method faces several challenges. Firstly, loads must be applied carefully to minimise the boundary layer effects. In addition, the cross-sectional properties calculated by BPE are sensitive to the length of the blade segment that has been chosen to perform the finite-element analysis. Decreasing the length of the blade segment may even result in a singular stiffness matrix under some extreme situations, such as a blade having highly twisted or considerably distorted cross sections [118].

\subsection{D FEM based model}

Several other cross-sectional analysis tools based on 2D FEM have also been developed. Cesnik and Hodges [119] developed VABS (Variational Asymptotic Beam Sectional analysis) based on a variational asymptotic method, in which the 3D structural model is replaced with a $2 \mathrm{D}$ model in terms of an asymptotic series of several small parameters of the structure. VABS needs a 2D finite-element discretisation of the cross section to yield its input files, which are the 2D mesh of the cross section and the corresponding materials. For a realistic wind turbine blade made of layers of composites, generating VABS input files is very tedious and requires a separate pre-processor called PreVABS [120]. Blasques [121] developed a cross-sectional analysis tool named BECAS (BEam Cross section Analysis Software) based on anisotropic beam theory, which was originally presented by Giavotto et al. [122] for calculating the stiffness and stresses of a beam section made of anisotropic and non-homogeneous materials. Similar to VABS, BECAS also needs a 2D finiteelement discretisation of the cross section. A separate pre-processor called Aifoil2BECAS [123], which is a Python code, is employed to generate the input files for BECAS. Fig. 21 from Ref. [123] 
depicts an example of 2D mesh generated by Airfoil2BECAS. Currently, the cross section in Airfoil2BECAS is restricted to eight distinct regions, to which thickness and layup information can be assigned.

Compared to the 3D FEM-based cross-sectional analysis models, the 2D FEM-based crosssectional analysis models require fewer computational resources, but they are still not efficient enough because they require a separate pre-processer to generate their input files.

\subsection{CLT (classical lamination theory) based model}

In comparison to the finite-element techniques, CLT [124], which is an extension of the classical plate theory [125] to laminated plates, is efficient and reasonably accurate. CLT can be utilised to combine properties and the angle of each ply in a pre-defined stacking sequence to determine the overall effective performance for a laminated structure. Based on some reasonable assumptions (such as linear strain and plane stress), CLT transfers a complex 3D elasticity problem into a solvable 2D problem [126].

CLT has been extensively utilised for analysing the structural performance of composite materials $[127,128]$. In terms of wind turbine composite blades, Bir [129, 130] developed a Fortran code called PreComp (Pre-processor for computing Composite blade properties) at NREL (National Renewable Energy Laboratory) based on CLT. PreComp does not require a separate pre-processor to create the input files, which are the geometric shape and interior structural layout of the blades, and allows a general layup of composite laminates and an arbitrary number of shear webs. However, PreComp ignores the effects of shear webs in calculating torsional stiffness. In other words, if the number of shear webs on a cross section is altered, no change in the calculation results of torsional stiffness will be observed using PreComp. This is invalid for a realistic blade cross section, where the torsional stiffness will be increased as the number of shear webs grows. Authors [131] developed a Matlab code called CBCSA (Composite Blade Cross Section Analysis) by incorporating CLT with the EBSFT (extended Bredt-Batho shear flow theory). In CBCSA, all cross-sectional laminates are discretised into many area segments, each of which encloses several angled plies. The effective engineering constants of each angled ply are calculated using CLT. The area moments of inertia of each area segment are first calculated with respect to their local axes and centroid, and then transformed to the elastic axes and centre of the cross section. The torsional stiffness is calculated using EBSFT while the other cross-sectional properties are obtained by means of adding the contributions of all the area segments. The flowchart of CBCSA is presented in Fig. 22 from Ref. [131]. CBCSA can rapidly calculate the cross-sectional properties of the composite blades, and its results have been validated against experimental data. CBCSA provides 
a more accurate torsional stiffness calculation than PreComp due to the consideration of the shear web effects by using EBSFT.

Compared to both the 3D FEM-based and 2D FEM-based cross-sectional analysis models, the CLT based cross-sectional analysis models are more efficient. The drawback of CLT-based models is that they ignore the shear deformation in calculating cross-sectional properties, making them unsuitable for modelling short and thick blades.

\section{Current implementations}

Investigating the aeroelasticity of wind turbine blades needs a wind-turbine-specific aeroelastic model. One of the earliest wind turbine aeroelastic models, STALLVIB [132], was developed within the European Non-Nuclear Energy project JOULE III. This model was developed for predicting dynamic loads and investigating the edgewise instability problems.

After the first attempts, a considerable number of aeroelastic models have since been developed. The models being widely used within wind turbine research organisations and industrial practices are listed below with short descriptions.

- $\quad$ ADAMS/WT (Automatic Dynamic Analysis of Mechanical Systems - Wind Turbine)

- ADAMS/WT is a wind-turbine-specific add-on for ADAMS, which is a widely used commercial multi-body dynamics software package. ADAMS/WT has been developed by MDI (Mechanical Dynamics Inc.) with the help of NREL (National Renewable Energy Laboratory) [133].

- FAST (Fatigue, Aerodynamics, Structures, and Turbulence)

- FAST has been developed by the NREL to model both two- and three-bladed horizontal-axis wind turbines. In 2005, GL (Germanischer Lloyd), one of the leading certification organisations in the wind energy area, issued FAST with a certification on its load calculation of onshore wind turbines [134].

\section{- $\quad$ FLEX5}

- FLEX5 has been developed by the Fluid Mechanics Department at the DTU (Technical University of Denmark). This code is capable of simulating wind turbines with different configurations, e.g. turbines with one to three blades [112] 
- GAST (General Aerodynamic and Structural Prediction Tool for Wind Turbines)

- GAST has been developed by the National Technical University of Athens. The code contains an additional module to generate turbulent wind fields and a postprocessing module to perform fatigue analysis [135].

- GH-Bladed

- GH-Bladed is an integrated commercial software package developed by GH (Garrad Hassan) Ltd. GH-Bladed has a friendly windows-based GUI (graphical user interface), and has been validated against experimental data for a number of wind turbines with different sizes and configurations [136].

- HAWC2 (Horizontal Axis Wind Turbine Code $2^{\text {nd }}$ generation)

- HAWC2 has been developed by the DTU. The code analyses the aeroelastic behaviour of horizontal axis wind turbines in a time domain [137].

- $\quad$ PHATAS (Program for Horizontal Axis Wind Turbine Analysis Simulation)

- PHATAS has been developed by ECN (Energy research Centre of the Netherlands) for predicting the dynamic behaviour and the corresponding loads on horizontal axis wind turbines. PHATAS includes additional programs used to generate load-case files following international standard IEC or GL [138].

The features of the above seven aeroelastic models are summarised in Table 1. From Table 1 we can see that six of the seven aeroelastic models use BEM (blade element momentum) theory as the aerodynamic part. For the structural part, all of these models represent wind turbine blades as a series of 1D beam elements, and require blade cross-sectional properties as input. All the three discretisation methods, i.e. modal approach, MBD (multi-body dynamics) and 1D FEM (finiteelement method), which are addressed in Section 3.2, find applications in these aeroelastic models.

Additionally, most of these aeroelastic models are linear models based on assumption of small blade deflections, and do not take account of large deflection effects on modelling responses and loads. However, with the increasing size and flexibility of large wind turbine blades, this assumption is not valid anymore because the blades often experience large deflections, which introduce significant geometric nonlinearities. Therefore, developing nonlinear aeroelastic models to take account of geometric nonlinearities is necessary for future development of large wind turbine blades. 


\section{Discussion}

The key components for aeroelastic modelling of wind turbine blades are aerodynamic, structural and cross-sectional analysis models. A discussion of these models is presented below.

\subsection{Aerodynamic model}

For the aerodynamic part of aeroelastic modelling of wind turbine blades, four types of aerodynamic models are generally employed, i.e. BEM (blade element momentum) model, vortex model, actuator type model and CFD (computational fluid dynamics) model. Compared to other aerodynamic models, the BEM model is the fastest and capable of providing accurate results when reliable airfoil aerodynamic data are available. For this reason, the BEM model has been employed as the aerodynamic part of most wind turbine aeroelastic models. In order to better understand wake dynamics, the vortex model has been applied to wind turbine blades. Compared to the BEM model, the vortex model requires more computational resources, and viscous effects are ignored in the vortex model, which limits its application to wind turbines to some extent. The actuator type model should be credited because it provides a better insight into the flow development and contributes to a better understanding of the wake dynamics. However the actuator type model demands more computational resources than the BEM model, and it does not predict aerodynamic loads more accurately than the BEM model because it still relies on tabulated airfoil aerodynamic data for the calculation of aerodynamic loads on the blades. The CFD model solves the governing equations of fluid flow at thousands of positions on and around the blade in an iterative process, and does not need the predetermined airfoil aerodynamic data for the calculation of aerodynamic loads on the blades. It is capable of modelling complex 3D flow fields accurately and simulating fluid dynamics reliably. Currently, CFD is computationally too expensive, which is the main obstacle for its industrial application in aeroelastic modelling. Table 2 presents a comparison of the four types of aerodynamic models in terms of accuracy, computational speed, whether requiring airfoil aerodynamic data and whether including viscous effects.

\subsection{Structural model}

For the structural part of aeroelastic modelling of wind turbine blades, the blade structure can be modelled using either a 3D FEM model with shell elements or a 1D beam model with beam elements. The 3D FEM is an incredible tool for examining the detailed stress distribution within a blade. However, the 3D FEM is computationally expensive and this undesirable feature limits its application in aeroelastic modelling to some extent. Compared to the 3D FEM model, the 1D beam model is much faster and is capable of providing accurate results if constructed properly. 
Therefore almost all the aeroelastic codes model the structure of a wind turbine blade as a series of $1 \mathrm{D}$ beam elements instead of 3D shell elements.

In order to discretise the blade into a series of 1D beam elements, three discretisation methods are often used in aeroelastic modelling of wind turbine blades, i.e. modal approach, MBD (multi-body dynamics) and 1D FEM (finite-element method). Modal approach is computationally efficient and is capable of providing reasonable results in cases of small deflections. However, the flexibility of modal approach is limited somewhat by its constraints on the type and number of DOFs (degree of freedoms) allowed in the structure. Additionally, the accuracy of the modal approach greatly relies on the prescribed mode shapes, which are generally obtained by a finite-element-based preprocessor. The MBD method, which discretises the structure into a number of bodies, benefits from high modelling flexibility due to its capability to generate and couple together an arbitrary number of separate bodies in a single dynamic system. Compared to the modal approach, the MBD method requires more computational resources, but it enables an increased number of DOFs to be modelled. Compared to above two discretisation methods, 1D FEM allows a more comprehensive and accurate deformation description of wind turbine blades, and requires only slightly more computational resources than other two discretisation methods. Additionally, it enables a large number of DOFs to be modelled. Table 3 presents a comparison of the three types of discretisation methods in terms of accuracy, computational speed, number of DOFs and whether requiring prescribed modal shapes.

\subsection{Cross-sectional analysis model}

To construct the 1D beam model of a wind turbine blade for aeroelastic modelling, the crosssectional properties of the blade, such as mass per unit length and sectional stiffness, are essential information. Due to the intrinsic nature of composite materials and the complexity of blade structural topologies, obtaining the cross-sectional properties of a wind turbine blade is quite challenging.

In order to obtain the cross-sectional properties of a wind turbine blade, various cross-sectional analysis models have been developed, which can be categorised into three groups, i.e. 3D FEMbased model, 2D FEM-based model and CLT-based model. 3D FEM-based models are computationally expensive because they rely on computationally complicated post-processing of force-displacement data. Compared to the 3D FEM-based models, 2D FEM-based models requires fewer computational resources, but they are not efficient enough since they require a separate preprocessor to generate the input files. CLT-based models are most efficient, however shear deformations of cross sections are ignored in the processing the cross-sectional properties, which makes them unsuitable for modelling short and thick blades. Further theoretical and experimental 
works are required to validate these cross-sectional analysis models. Table 4 presents a comparison of the three types of cross-sectional analysis models in terms of accuracy, computational speed, whether requiring post-processing of force displacement data and whether requiring a preprocessor.

\section{Conclusions}

Ever increasing size and flexibility of large wind turbine blades have been introducing severer aeroelastic effects, which are caused by the interaction of aerodynamic loads, elastic deflections and inertial dynamics. The aeroelastic effects may cause aeroelastic instability problems, such as edgewise instability and flutter, which can be devastating both to the blades and wind turbine when the wind turbine is getting larger and larger. It is therefore crucial to investigate the aeroelasticity characterisation of wind turbine blades for next generation of large wind turbine development.

This paper presents a comprehensive review on the aeroelastic modelling of wind turbine blades, covering aerodynamic models, structural models and cross-sectional analysis. The advantages and disadvantages of each model are critically analysed, and the state-of-the-art implementations are presented.

The BEM (blade element momentum) and 1D beam models have been used in most aeroelastic models due to their high efficiency and reasonable accuracy in computation. However, these models are incapable of providing detailed aerodynamic information (such as visualisation of flow field around the blade) and detailed structural information (such as stress distributions within each layer of composite blades). Although CFD (computational fluid dynamics) and 3D FEM (finite element method) models are computationally much expensive than those simple models, coupling CFD and 3D FEM is one of the future trends for aeroelastic modelling in order to obtain more precise results for complex cases and to gain detailed information for blade designers to further optimise the blade.

Combining CFD with simpler aerodynamic models to develop hybrid aerodynamic models (such as hybrid CFD/BEM model and hybrid CFD/vortex model) provides the possibility to well utilise the advantages of both CFD and the simpler model, retaining desirable accuracy and saving computational time. More studies are to be carried out in developing hybrid aerodynamic models and applying these models to aeroelastic modelling.

Additionally, the majority of existing aeroelastic models are linear models based on assumption of small blade deflections. However, with the increasing size and flexibility of large wind turbine 
blades, this assumption is not valid anymore because the blades often experience large deflections, which introduce significant geometric nonlinearities. Therefore, developing nonlinear aeroelastic models to take account of geometric nonlinearities would also be the future trend of aeroelastic modelling of wind turbine blades.

\section{References}

[1] Grasso F. Investigation of Wind Turbine Rotor Concepts for Offshore Wind Farms. Journal of Physics: Conference Series: IOP Publishing; 2014. p. 012032.

[2] Jamieson P, Branney M. Structural Considerations of a 20MW Multi-Rotor Wind Energy System. Journal of Physics: Conference Series: IOP Publishing; 2014. p. 012013.

[3] Ederer N. The right size matters: Investigating the offshore wind turbine market equilibrium. Energy. 2014;68:910-21.

[4] Moeller T. Blade cracks signal new stress problem. WindPower Monthly. 1997;25.

[5] Glauert H. Airplane propellers. Aerodynamic theory. 1935;4:169-360.

[6] McGowan JG, Rogers AL. Wind Energy Explained: Theory, Design and Application: John Wiley \& Sons; 2010.

[7] Liu X, Wang L, Tang X. Optimized linearization of chord and twist angle profiles for fixedpitch fixed-speed wind turbine blades. Renewable Energy. 2013;57:111-9.

[8] Vries O. Fluid dynamic aspects of wind energy conversion. DTIC Document; 1979.

[9] Xu G, Sankar LN. Application of a viscous flow methodology to the NREL phase VI rotor. ASME 2002 Wind Energy Symposium: American Society of Mechanical Engineers; 2002. p. 8393.

[10] Hand MM, Simms D, Fingersh L, Jager D, Cotrell J. Unsteady aerodynamics experiment phase VI: test configuration and available data campaigns: National Renewable Energy Laboratory; 2001.

[11] Glauert $\mathrm{H}$. The analysis of experimental results in the windmill brake and vortex ring states of an airscrew: HM Stationery Office; 1926.

[12] Moriarty PJ, Hansen AC. AeroDyn theory manual: National Renewable Energy Laboratory Golden, Colorado, USA; 2005.

[13] Buhl ML. A new empirical relationship between thrust coefficient and induction factor for the turbulent windmill state: National Renewable Energy Laboratory Golden, CO; 2005.

[14] Bossanyi E. GH Bladed-Theory Manual, Version 3.81. Garrad Hassan and Partners. 2008:16.

[15] Burton T, Sharpe D, Jenkins N, Bossanyi E. Wind energy handbook: John Wiley \& Sons; 2001.

[16] Spera D. Wind turbine technology-fundamental concepts of wind turbine technology. ASME Press, New York; 1998.

[17] Tang X. Aerodynamic design and analysis of small horizontal axis wind turbine blades: University of Central Lancashire; 2012.

[18] Hansen MOL, Sørensen JN, Voutsinas S, Sørensen N, Madsen HA. State of the art in wind turbine aerodynamics and aeroelasticity. Progress in aerospace sciences. 2006;42:285-330.

[19] Øye S. Tjæreborg wind turbine (Esbjerg) Structural dynamics data. VK-186, Dept of Fluid Mechanics, Technical University of Denmark: Lyngby. 1991.

[20] Øye S. Tjæreborg wind turbine: Dynamic flow measurement. AFM Notat VK. 1992;233.

[21] Pitt DM, Peters DA. Theoretical prediction of dynamic-inflow derivatives. Vertica.

1981;5:21-34.

[22] Snel H, Schepers J, Nederland SEC. Joint investigation of dynamic inflow effects and implementation of an engineering method: Netherlands Energy Research Foundation ECN; 1995. [23] Henriksen LC, Hansen MH, Poulsen NK. A simplified dynamic inflow model and its effect on the performance of free mean wind speed estimation. Wind Energy. 2013;16:1213-24.

[24] Chen X, Agarwal R. Inclusion of a simple dynamic inflow model in the blade element momentum theory for wind turbine application. Journal homepage: www IJEE IEEFoundation org. 2014;5:183-96. 
[25] Zhang P, Huang S. Review of aeroelasticity for wind turbine: Current status, research focus and future perspectives. Frontiers in Energy. 2011;5:419-34.

[26] Theodorsen T. General theory of aerodynamic instability and the mechanism of flutter. 1949.

[27] Pierce KG. Wind turbine load prediction using the beddoes-leishman model for unsteady aerodynamics and dynamic stall: The University of Utah; 1996.

[28] Leishman J, Beddoes T. A Semi-Empirical Model for Dynamic Stall. Journal of the American Helicopter Society. 1989;34:3-17.

[29] Tran C, Petot D. Semi-empirical model for the dynamic stall of airfoils in view of the application to the calculation of responses of a helicopter blade in forward flight. 1980.

[30] Snel H. Heuristic modelling of dynamic stall characteristics. EWEC-CONFERENCE-: BOOKSHOP FOR SCIENTIFIC PUBLICATIONS; 1997. p. 429-33.

[31] Øye S. Dynamic stall-simulated as time lag of separation. Proceedings of the 4th IEA Symposium on the aerodynamics of wind turbines 1991.

[32] Tarzanin F. Prediction of control loads due to blade stall. Journal of the American Helicopter Society. 1972;17:33-46.

[33] Hansen MH, Gaunaa M, Aagaard Madsen H. A Beddoes-Leishman type dynamic stall model in state-space and indicial formulations2004.

[34] Holierhoek J, De Vaal J, Van Zuijlen A, Bijl H. Comparing different dynamic stall models. Wind Energy. 2013;16:139-58.

[35] Larsen JW, Nielsen SR, Krenk S. Dynamic stall model for wind turbine airfoils. Journal of Fluids and Structures. 2007;23:959-82.

[36] Wang Q, Xu Y, Xu J. A New Stall Delay Model for HAWT Based on Inviscid Theory.

Fluid-Structure-Sound Interactions and Control: Springer; 2014. p. 363-8.

[37] Dowler JL, Schmitz S. A solution-based stall delay model for horizontal-axis wind turbines. Wind Energy. 2014.

[38] Dumitrescu H, Frunzulică F, Cardoş V. Improved stall-delay model for horizontal-axis wind turbines. Journal of Aircraft. 2012;50:315-9.

[39] Lanzafame R, Messina M. BEM theory: How to take into account the radial flow inside of a 1-D numerical code. Renewable Energy. 2012;39:440-6.

[40] Chaney K, Eggers AJ, Moriarty PJ, Holley WE. Skewed wake induction effects on thrust distribution on small wind turbine rotors. Journal of solar energy engineering. 2001;123:290-5.

[41] Ning S, Hayman G, Damiani R, Jonkman J. Development and Validation of a New Blade

Element Momentum Skewed-Wake Model within AeroDyn: Preprint. National Renewable Energy Laboratory (NREL), Golden, CO.; 2014.

[42] Tangler J. Comparison of wind turbine performance prediction and measurement. Journal of solar energy engineering. 1982;104:84-8.

[43] Lanzafame R, Messina M. Fluid dynamics wind turbine design: Critical analysis, optimization and application of BEM theory. Renewable energy. 2007;32:2291-305.

[44] Vaz JRP, Pinho JT, Mesquita ALA. An extension of BEM method applied to horizontal-axis wind turbine design. Renewable Energy. 2011;36:1734-40.

[45] Kolios A, Chahardehi A, Brennan F. Experimental determination of the overturning moment and net lateral force generated by a novel vertical axis wind turbine: experiment design under load uncertainty. Experimental Techniques. 2013;37:7-14.

[46] Wang L, Tang X, Liu X. Optimized chord and twist angle distributions of wind turbine blade considering Reynolds number effects. Internation conference on Wind Energy: Materials, Engineering and Policies (WEMEP). Bits Pilani Hyderabad Campus (India)2012.

[47] Wang L, Tang X, Liu X. Blade design optimisation for fixed-pitch fixed-speed wind turbines. International Scholarly Research Notices. 2012;2012.

[48] Zhao J, Liu XW, Wang L, Tang XZ. Design attack angle analysis for fixed-pitch variablespeed wind turbine. Advanced Materials Research. 2012;512:608-12.

[49] Liu X, Wang L, Tang X. Investigation on the impact of design wind speed and control strategy on the performance of fixed-pitch variable-speed wind turbines.

[50] Chattot J-J. Helicoidal vortex model for wind turbine aeroelastic simulation. Computers \& structures. 2007;85:1072-9.

[51] Qiu Y-X, Wang X-D, Kang S, Zhao M, Liang J-Y. Predictions of unsteady HAWT aerodynamics in yawing and pitching using the free vortex method. Renewable Energy. 2014;70:93-106. 
[52] Breton SP, Coton FN, Moe G. A study on rotational effects and different stall delay models using a prescribed wake vortex scheme and NREL phase VI experiment data. Wind Energy. 2008;11:459-82.

[53] Gaunaa M, Sørensen NN, Døssing M. Prediction of steady aerodynamic performance of rotors with winglets using simple prescribed wake methods. 49th AIAA Aerospace Sciences Meeting and Exhibit2011.

[54] Riziotis V, Manolas D, Voutsinas S. Free-wake Aeroelastic Modelling of Swept Rotor Blades. In: EWEA, editor.2011.

[55] Jeong M-S, Yoo S-J, Lee I. Wind Turbine Aerodynamics Prediction Using Free-wake Method in Axial Flow. International Journal of Modern Physics: Conference Series: World Scientific; 2012. p. 166-72.

[56] Chkir S. Unsteady Loads Evaluation for a Wind Turbine Rotor using free wake method. Energy Procedia. 2011;6:777-85.

[57] Jeong M-S, Kim S-W, Lee I, Yoo S-J, Park K. The impact of yaw error on aeroelastic characteristics of a horizontal axis wind turbine blade. Renewable Energy. 2013;60:256-68.

[58] Rankine WJM, Architects IoN. On the mechanical principles of the action of propellers 1865. [59] Froude R. On the part played in propulsion by differences of fluid pressure. Transactions of the Institute of Naval Architects. 1889;30:390-405.

[60] Shen WZ, Hansen MOL, Sørensen JN. Determination of the angle of attack on rotor blades. Wind Energy. 2009;12:91-8.

[61] SØRENSEN JN, Shen WZ. Numerical modeling of wind turbine wakes. Journal of fluids engineering. 2002;124:393-9.

[62] Shen WZ, Zhu WJ, Sørensen JN. Actuator line/Navier-Stokes computations for the MEXICO rotor: comparison with detailed measurements. Wind Energy. 2012;15:811-25.

[63] Shen WZ, Sørensen JN, Zhang J. Actuator surface model for wind turbine flow computations. 2007.

[64] Shen WZ, Zhang JH, Sørensen JN. The actuator surface model: a new Navier-Stokes based model for rotor computations. Journal of Solar Energy Engineering. 2009;131:011002.

[65] Sibuet Watters C, Masson C. Modeling of lifting-device aerodynamics using the actuator surface concept. International journal for numerical methods in fluids. 2010;62:1264-98.

[66] Kim T, Oh S, Yee K. Improved actuator surface method for wind turbine application. Renewable Energy. 2015;76:16-26.

[67] Dobrev I, Massouh F, Rapin M. Actuator surface hybrid model. IOP Publishing; 2007. p. 012019 .

[68] Imiela M, Wienke F, Rautmann C, Willberg C, Hilmer P, Krumme A. Towards Multidisciplinary Wind Turbine Design using High-Fidelity Methods. 33rd Wind Energy Symposium Kissimmee, Florida 5 - 9 January 2015.

[69] Planchard D. SolidWorks 2014 Reference Guide: SDC Publications; 2014.

[70] Unigraphics N. User Documentation. Unigraphics Solutions, Cypress, CA. 2014.

[71] Bijl H, Van Zuijlen A, Van Mameren A. Validation of adaptive unstructured hexahedral mesh computations of flow around a wind turbine airfoil. International journal for numerical methods in fluids. 2005;48:929-45.

[72] Van Zuijlen A, Bijl H, Dufour G, Van Mameren A. Evaluation of adaptive, unstructured CFD calculations of the flow around the DU91 airfoil. 2003.

[73] Carrigan TJ, Dennis BH, Han ZX, Wang BP. Aerodynamic shape optimization of a verticalaxis wind turbine using differential evolution. ISRN Renewable Energy. 2012;2012.

[74] Quarteroni A. Navier-Stokes equations. Numerical Models for Differential Problems: Springer; 2014. p. 429-82.

[75] Sanderse B. Aerodynamics of wind turbine wakes. Energy Research Center of the

Netherlands (ECN), ECN-E-09-016, Petten, The Netherlands, Tech Rep. 2009;5:153.

[76] Quartapelle L. Numerical solution of the incompressible Navier-Stokes equations: Birkhäuser; 2013.

[77] ANSYS C. User Manual, ANSYS CFX. Inc, Canonsburg, PA. 2012.

[78] Huebner KH, Dewhirst DL, Smith DE, Byrom TG. The finite element method for engineers: John Wiley \& Sons; 2008.

[79] Menter FR. Improved two-equation k-omega turbulence models for aerodynamic flows.

NASA STI/Recon Technical Report N. 1992;93:22809. 
[80] Lam C, Bremhorst K. A modified form of the k- $\varepsilon$ model for predicting wall turbulence. Journal of Fluids Engineering. 1981;103:456-60.

[81] SPALART P, ALLMARAS S. A one-equation turbulence model for aerodynamic flows. 1992.

[82] Kalvig S, Manger E, Hjertager B. Comparing different CFD wind turbine modelling approaches with wind tunnel measurements. Journal of Physics: Conference Series: IOP Publishing; 2014. p. 012056.

[83] Cakmakcioglu S, Sert I, Tugluk O, Sezer-Uzol N. 2-D and 3-D CFD Investigation of NREL S826 Airfoil at Low Reynolds Numbers. Journal of Physics: Conference Series: IOP Publishing; 2014. p. 012028.

[84] Shen WZ, Zhu WJ, Sørensen JN. Study of tip loss corrections using CFD rotor computations. Journal of Physics: Conference Series: IOP Publishing; 2014. p. 012094.

[85] Sagaut P, Drikakis D. Large Eddy Simulation. Encyclopedia of Aerospace Engineering. 2010. [86] Travin A, Shur M, Strelets M, Spalart P. Detached-eddy simulations past a circular cylinder. Flow, Turbulence and Combustion. 2000;63:293-313.

[87] Bechmann A, Sørensen NN, Zahle F. CFD Simulations of the MEXICO Rotor. Wind Energy. 2011;14:677-89.

[88] AbdelSalam AM, Ramalingam V. Wake prediction of horizontal-axis wind turbine using fullrotor modeling. Journal of Wind Engineering and Industrial Aerodynamics. 2014;124:7-19.

[89] Sanderse B, Pijl vdS, Koren B. Review of computational fluid dynamics for wind turbine wake aerodynamics. Wind Energy. 2011;14:799-819.

[90] Vermeer L, Sørensen JN, Crespo A. Wind turbine wake aerodynamics. Progress in aerospace sciences. 2003;39:467-510.

[91] Snel H. Review of aerodynamics for wind turbines. Wind energy. 2003;6:203-11.

[92] Hansen MO, Madsen HA. Review paper on wind turbine aerodynamics. Journal of fluids engineering. 2011;133:114001.

[93] Esfahanian V, Pour AS, Harsini I, Haghani A, Pasandeh R, Shahbazi A, et al. Numerical analysis of flow field around NREL Phase II wind turbine by a hybrid CFD/BEM method. Journal of Wind Engineering and Industrial Aerodynamics. 2013;120:29-36.

[94] Suzuki K, Schmitz S, Ouyang H, Chattot J-J. Extension of an Unsteady Hybrid NavierStokes/Vortex-Panel Solver to Wind Turbine Aerodynamic Simulations at Higher Yaw Angles. ASME Turbo Expo 2014: Turbine Technical Conference and Exposition: American Society of Mechanical Engineers; 2014. p. V03BT46A011-V03BT46A.

[95] Sturge D, Sobotta D, Howell R, While A, Lou J. A hybrid actuator disc-Full rotor CFD methodology for modelling the effects of wind turbine wake interactions on performance. Renewable Energy. 2015;80:525-37.

[96] Yu DO, Kwon OJ. Predicting wind turbine blade loads and aeroelastic response using a coupled CFD-CSD method. Renewable Energy. 2014;70:184-96.

[97] Bazilevs Y, Hsu MC, Kiendl J, Wüchner R, Bletzinger KU. 3D simulation of wind turbine rotors at full scale. Part II: Fluid-structure interaction modeling with composite blades.

International Journal for Numerical Methods in Fluids. 2011;65:236-53.

[98] Bazilevs Y, Hsu M-C, Scott M. Isogeometric fluid-structure interaction analysis with emphasis on non-matching discretizations, and with application to wind turbines. Computer Methods in Applied Mechanics and Engineering. 2012;249:28-41.

[99] Hsu M-C, Bazilevs Y. Fluid-structure interaction modeling of wind turbines: simulating the full machine. Computational Mechanics. 2012;50:821-33.

[100] Carrión M, Steijl R, Woodgate M, Barakos G, Munduate X, Gomez-Iradi S. Aeroelastic analysis of wind turbines using a tightly coupled CFD-CSD method. Journal of Fluids and Structures. 2014;50:392-415.

[101] Li Y, Castro A, Sinokrot T, Prescott W, Carrica P. Coupled multi-body dynamics and CFD for wind turbine simulation including explicit wind turbulence. Renewable Energy. 2015;76:33861.

[102] ANSYS. Version 15.0; ANSYS. Inc: Canonsburg, PA, USA November. 2013.

[103] ABAQUS. Version 6.12 Documentation ABAQUS/CAE User's Manual. 2013.

[104] Berg J, Resor B. Numerical manufacturing and design tool (NuMAD V2. 0) for wind turbine blades: User's guide. Sandia National Laboratories, Albuquerque, NM, Technical Report No SAND2012-728. 2012. 
[105] Verelst D. Flexible Wind turbine Blades: a BEM-FEM coupled model approach. Delft University of Technology (TU Delft). 2009.

[106] Buhl M. NWTC Design Codes(WT-Perf). Last modified. 2009.

[107] Bauchau O, Craig J. Euler-Bernoulli beam theory. Structural analysis: with applications to aerospace structures: Springer; 2009. p. 173-221.

[108] Oñate E. Timoshenko theory. Structural analysis with the finite element method Linear statics: volume 2: beams, plates and shells: Springer; 2013. p. 37-97.

[109] Hodges DH. Geometrically exact, intrinsic theory for dynamics of curved and twisted anisotropic beams. AIAA journal. 2003;41:1131-7.

[110] Wang L, Liu X, Renevier N, Stables M, Hall GM. Nonlinear aeroelastic modelling for wind turbine blades based on blade element momentum theory and geometrically exact beam theory. Energy. 2014.

[111] Mahri Z, Rouabah M. Fatigue estimation for a rotating blade of a wind turbine. Revue des énergies renouvelables. 2002;5:39-47.

[112] Øye S. FLEX 5 User Manual. Danske Techniske Hogskole. 1999.

[113] Shabana AA. Dynamics of multibody systems: Cambridge university press; 2013.

[114] Borg M, Collu M, Kolios A. Offshore floating vertical axis wind turbines, dynamics modelling state of the art. Part II: Mooring line and structural dynamics. Renewable and Sustainable Energy Reviews. 2014;39:1226-34.

[115] Neto MA, Yu W, Ambrósio JA, Leal RP. Design Blades of a Wind Turbine Using Flexible Multibody Modelling. International Conference on Renewable Energies and Power Quality (ICREPQ'09), Valencia (Spain)2009. p. 2-3.

[116] Bir GS, Migliore PG. Preliminary structural design of composite blades for two-and threeblade rotors: National Renewable Energy Laboratory; 2004.

[117] David M, Daniel L. Identification and Use of Blade Physical Properties. 43rd AIAA Aerospace Sciences Meeting and Exhibit: American Institute of Aeronautics and Astronautics; 2005.

[118] Malcolm DJ, Laird DL. Extraction of equivalent beam properties from blade models. Wind Energy. 2007;10:135-57.

[119] Cesnik CES, Hodges DH. VABS: a new concept for composite rotor blade cross-sectional modeling. Journal of the American Helicopter Society. 1997;42:27-38.

[120] Chen H, Yu W. Manual of PreVABS. Utah State University; 2008.

[121] Blasques JP. User's Manual for BECAS. Technical University of Denmark; 2012.

[122] Giavotto V, Borri M, Mantegazza P, Ghiringhelli G, Carmaschi V, Maffioli G, et al. Anisotropic beam theory and applications. Computers \& Structures. 1983;16:403-13.

[123] Bitsche RD. Airfoil2BECAS: A preprocessor for the cross-section analysis software BECAS. Technical University of Denmark; 2012.

[124] Reddy JN. Mechanics of laminated composite plates and shells: theory and analysis: CRC; 2003.

[125] Timoshenko S, Woinowsky-Krieger S, Woinowsky S. Theory of plates and shells:

McGraw-hill New York; 1959.

[126] Jones RM. Mechanics of composite materials: CRC Press; 1998.

[127] Lee J, Lee S. Flexural-torsional behavior of thin-walled composite beams. Thin-walled structures. 2004;42:1293-305.

[128] Lee J, Kim SE, Hong K. Lateral buckling of I-section composite beams. Engineering Structures. 2002;24:955-64.

[129] Bir G. User's Guide to PreComp (Pre-Processor for Computing Composite Blade Properties). National Renewable Energy Laboratory (NREL); 2006.

[130] Bir G, Migliore P. Preliminary structural design of composite blades for two-and three-blade rotors. National Renewable Energy Laboratory; 2004.

[131] Wang L, Liu X, Guo L, Renevier N, Stables M. A mathematical model for calculating crosssectional properties of modern wind turbine composite blades. Renewable Energy. 2014;64:52-60. [132] Aagaard Madsen H, Thirstrup Petersen J, Björck A, Ganander H, Winkelaar D, Brand A, et al. Prediction of dynamic loads and induced vibrations in stall STALLVIB. Final report. Ris $\emptyset$ National Laboratory; 1998. 
[133] Elliott AS, Wright AD. ADAMS/WT: an industry-specific interactive modelling interface for wind turbine analysis. The Energy-Sources Technology Conference, New Orleans, LA, USA, 01/23-26/941994. p. 111-22.

[134] Jonkman JM, Buhl Jr ML. FAST user's guide. Golden, CO: National Renewable Energy Laboratory. 2005.

[135] S.G.Voutsinas VAVa. Gast: A general aerodynamic and structural prediction tool for wind turbines. European union wind energy conference. Dublin, Ireland1996.

[136] Bossanyi E. GH Bladed user manual. Garrad Hassan and Partners Ltd. 2009.

[137] Larsen T. How 2 HAWC2, the user's manual. RisøReport, Ris $\varnothing .2009$.

[138] Lindenburg C. PHATAS release'APR-2005'user's manual. Report ECN-I-05-005, ECN; 2005. 


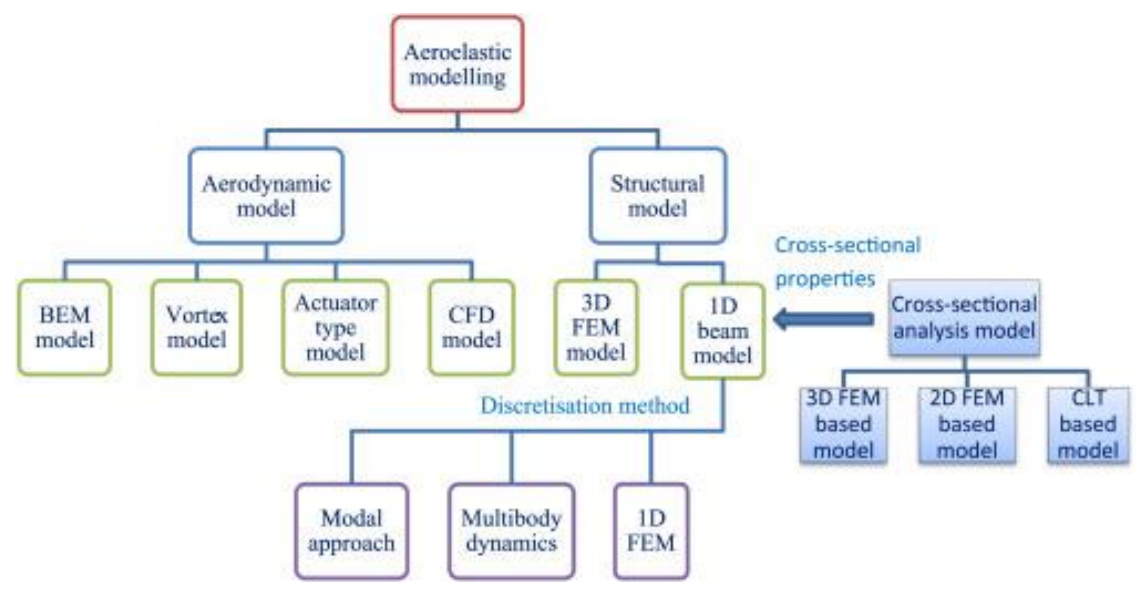

Figure 1. Components of the aeroelastic modelling of wind turbine blades 


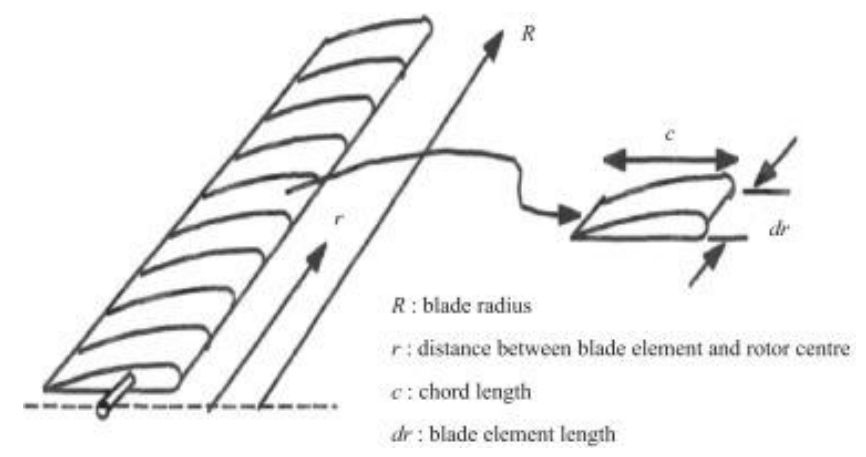

Figure 2. Schematic diagram of the blade element theory; reproduced from Ref. [6] 


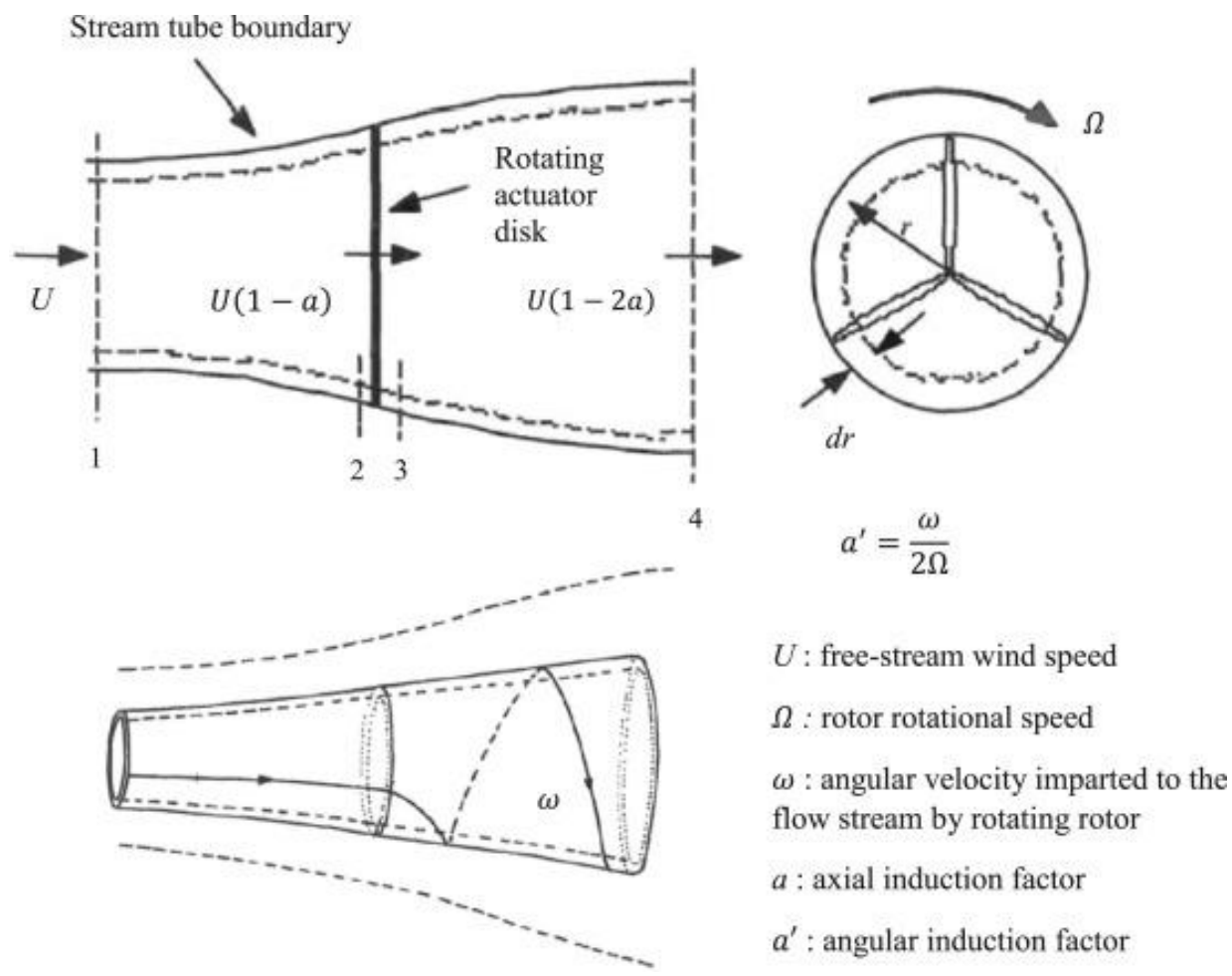

Figure 3. Schematic diagram of the blade momentum theory; reproduced from Ref. [6] 


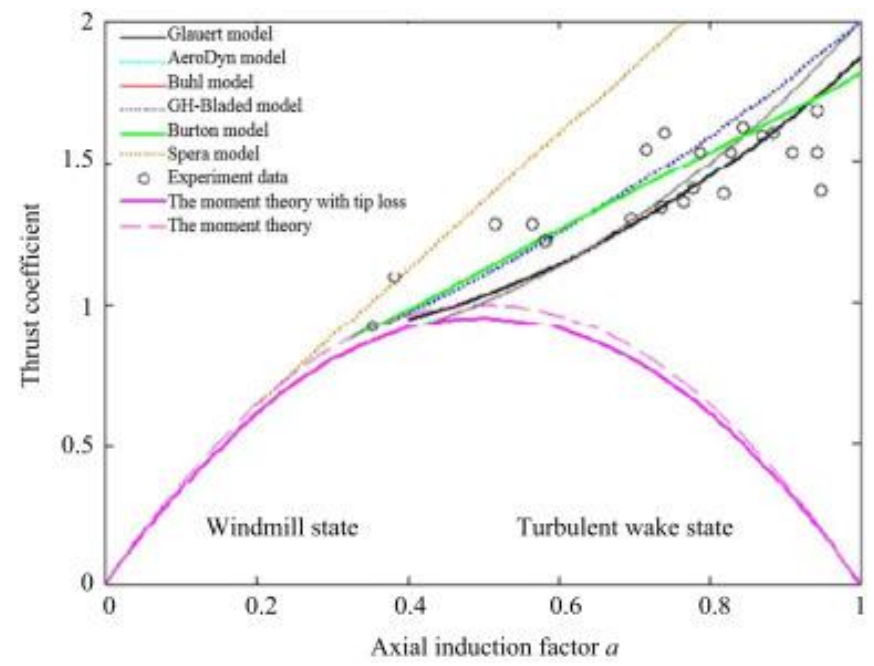

Figure 4. Comparison of thrust coefficient correction models; reproduced from Ref. [17] 


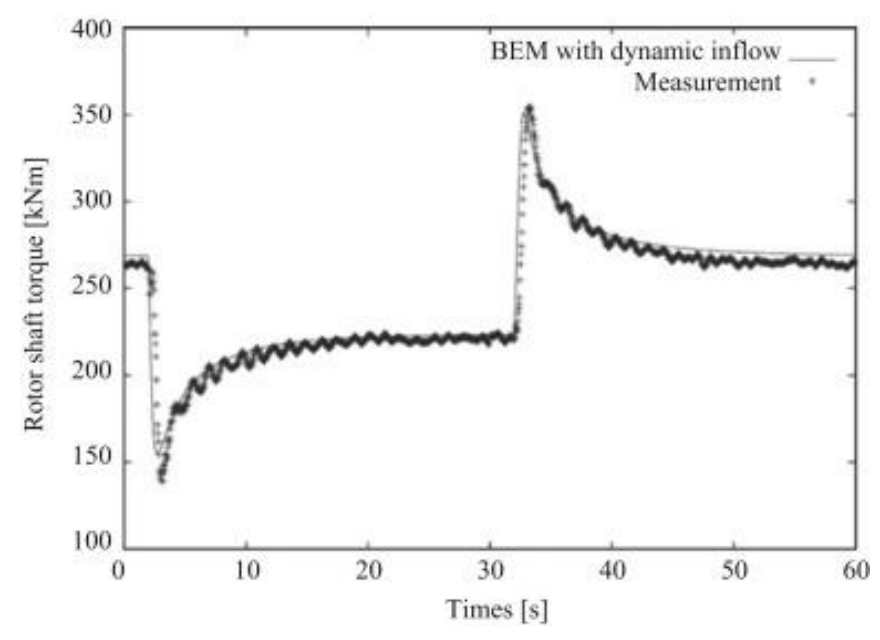

Figure 5. Predicted and measured dynamic response on the rotor shaft torque of the Tjaereborg $2 \mathrm{MW}$ wind turbine for a sudden change in the pitch angle; reproduced from Ref. [18] 


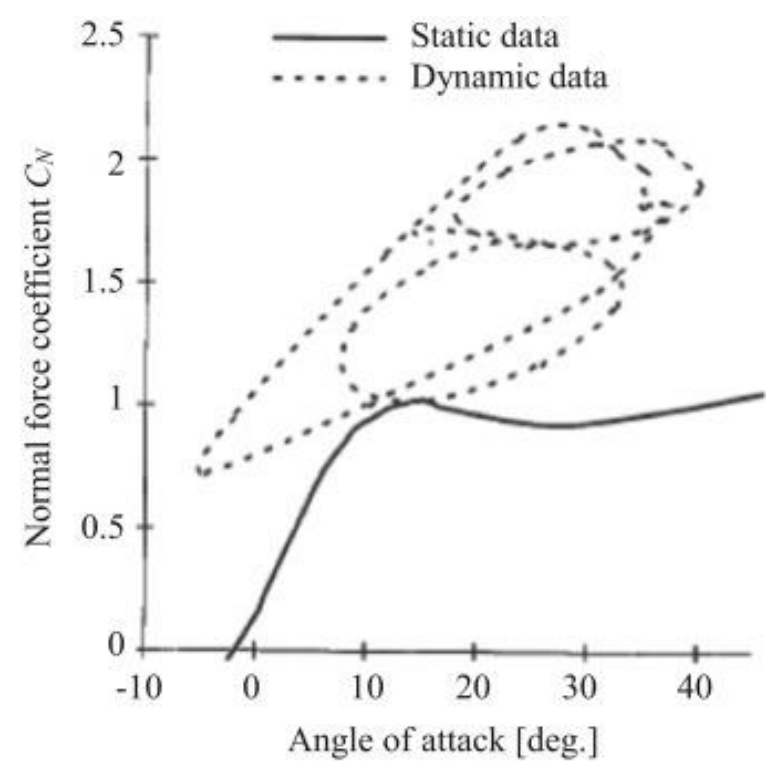

Figure 6. Dynamic stall event measured at the $30 \%$ span position of the CER; reproduced from Ref. [27] 


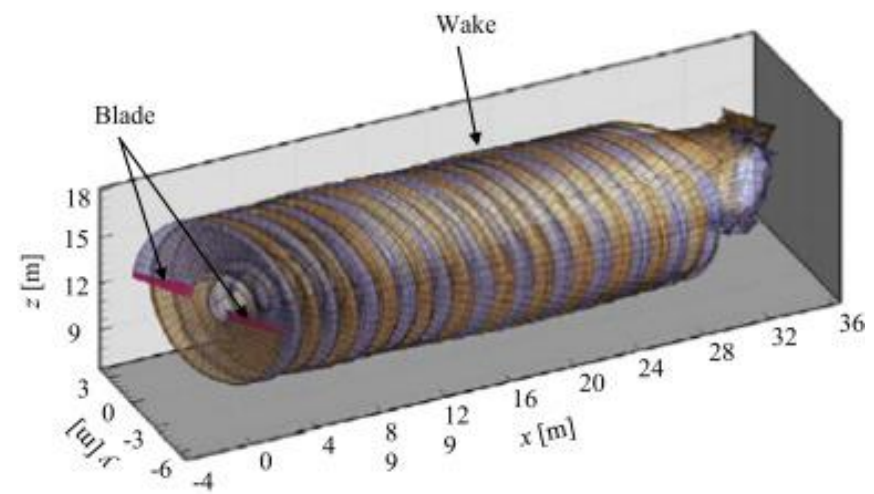

Figure 7. Wake modelling of a two-bladed wind turbine rotor using the vortex model; reproduced from Ref. [50] 


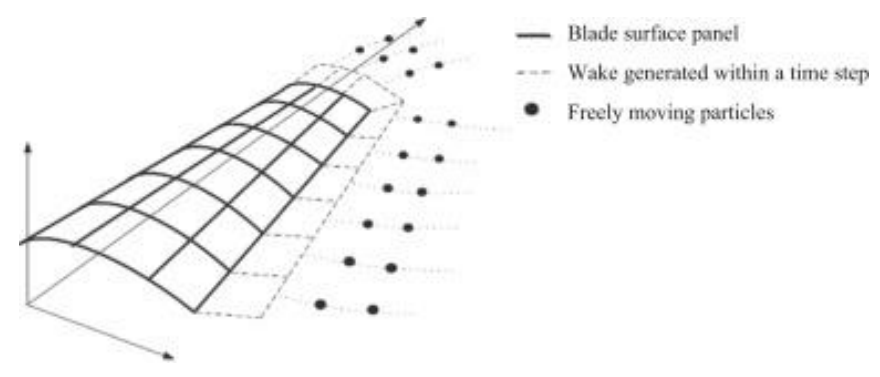

Figure 8. Typical layout of the free-wake modelling of a blade; reproduced from Ref.

[53] 


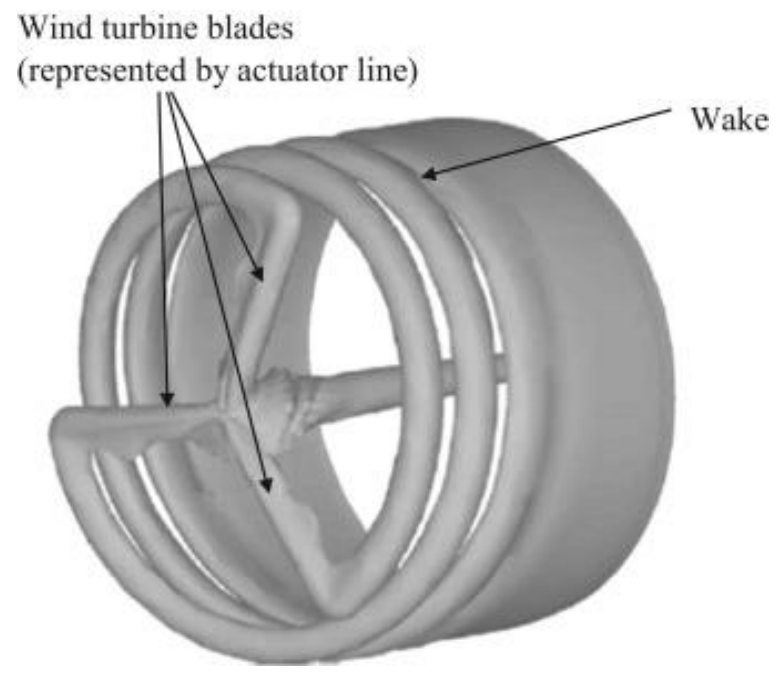

Figure 9. Wake modelling of a three-bladed wind turbine rotor based on the actuator line model; reproduced from Ref. [60] 


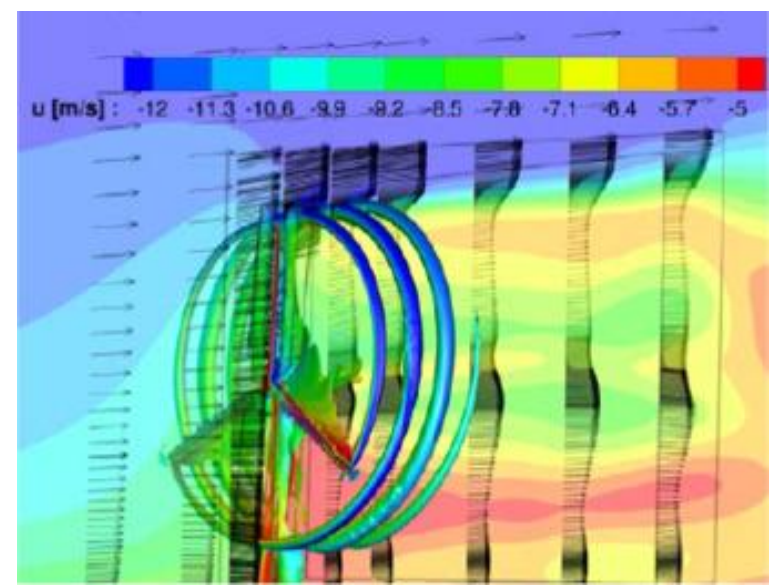

Figure 10. Visualised flow field around the blade using CFD [67] 


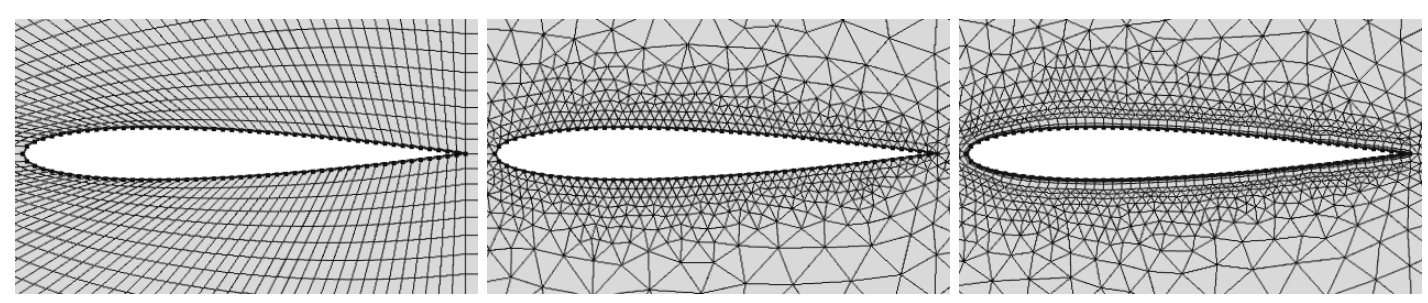
a. Structured mesh
b. Unstructured mesh
c. Hybrid mesh

Figure 11. The types of CFD meshes 


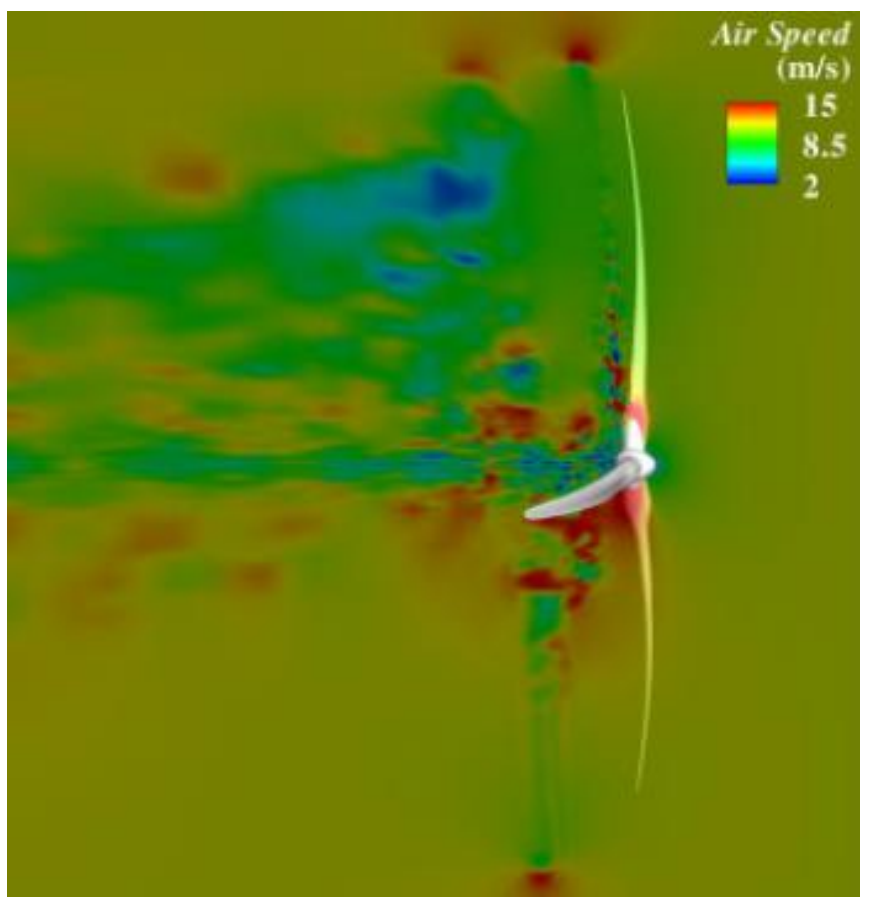

Figure 12. Air speed distribution around the deformed blade [85] 


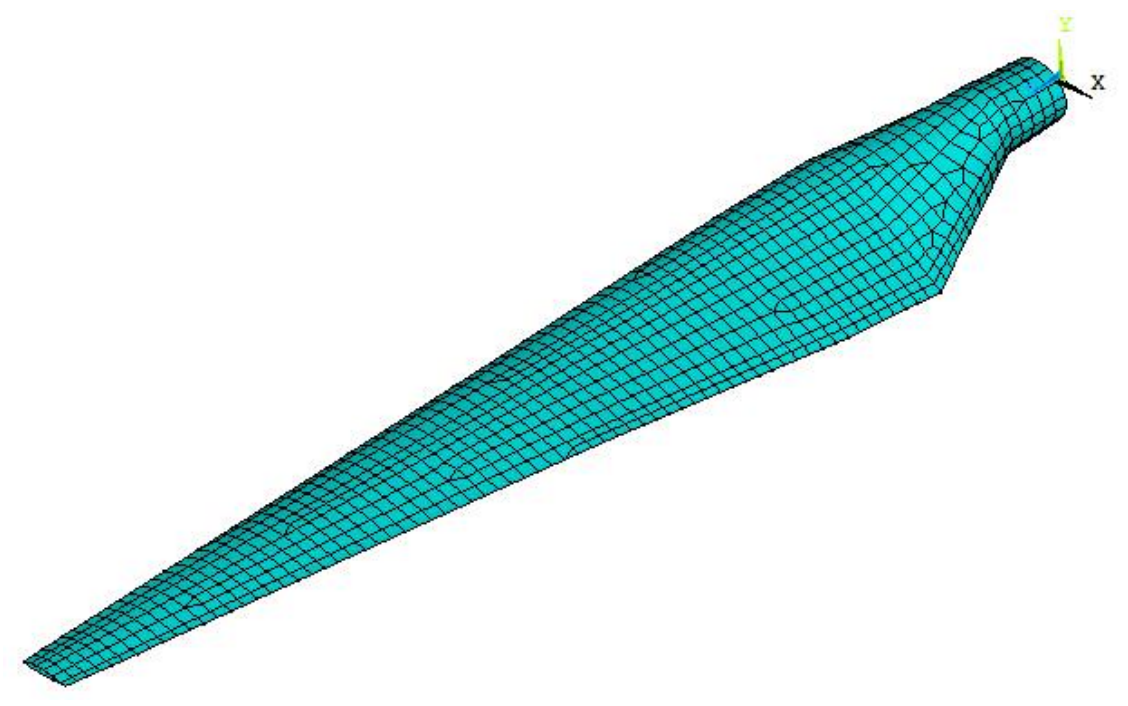

Figure 13. 3D FEM model of a wind turbine composite blade 


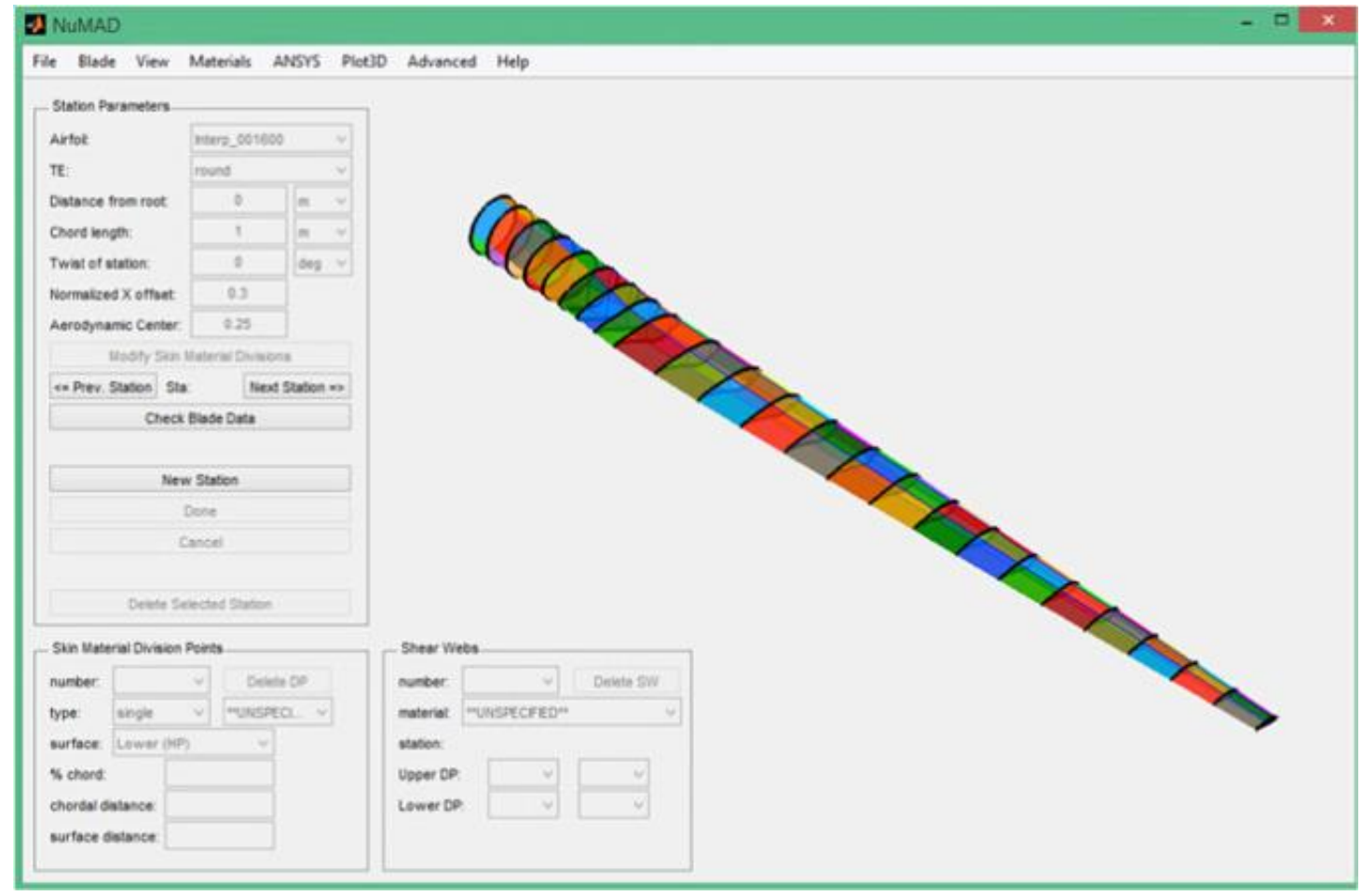

Figure 14. GUI of NuMAD v2.0 


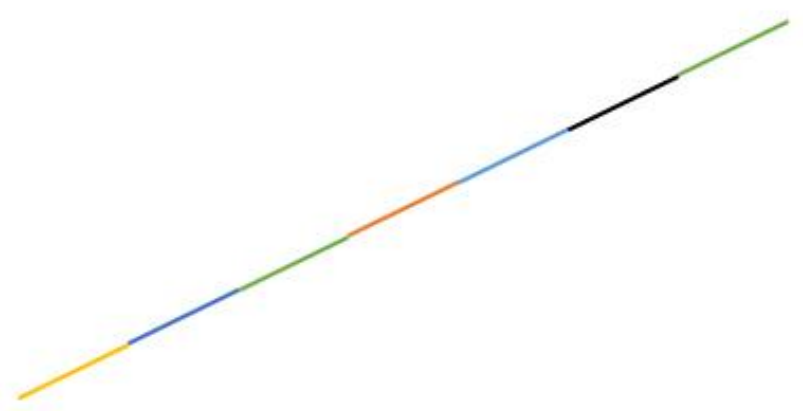

Figure 15. Examples of a beam model of wind turbine blades 

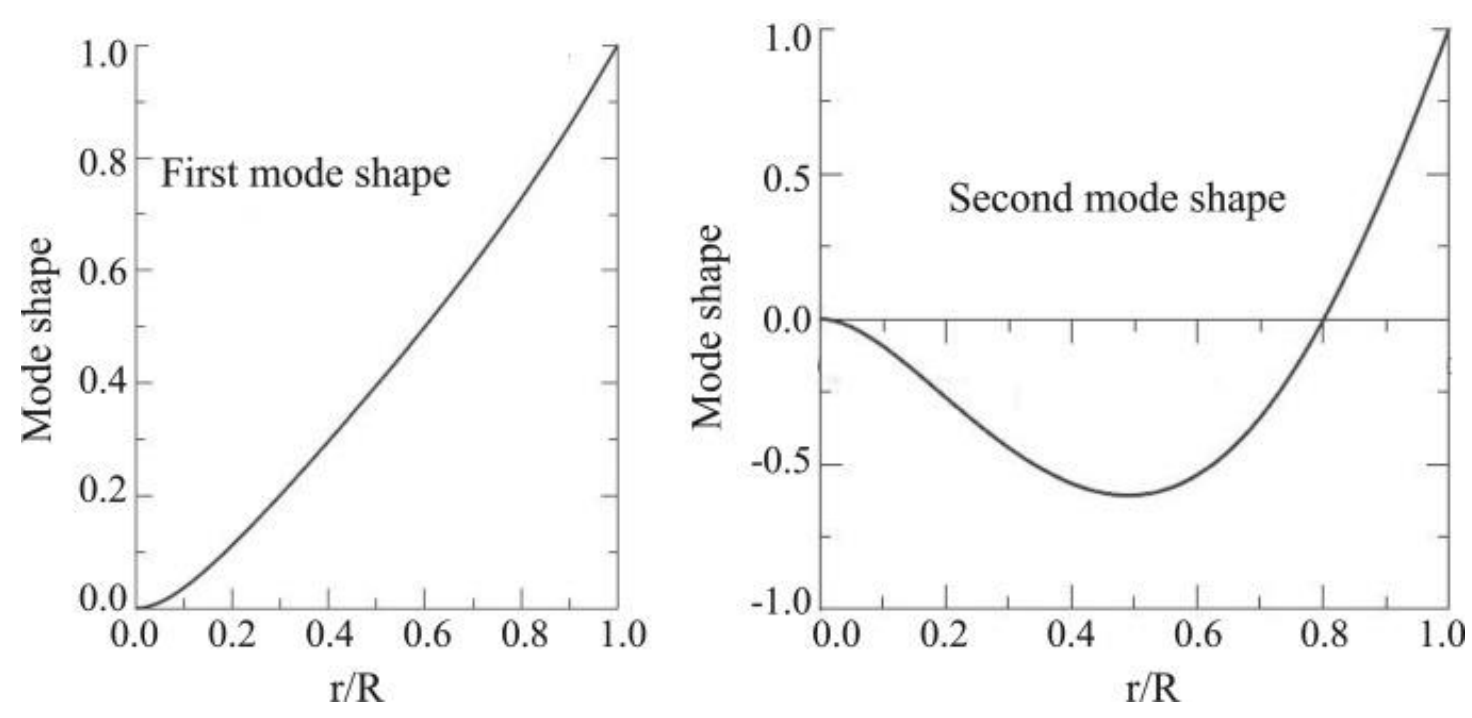

Figure 16. First and second mode shape of a wind turbine blade; reproduced from Ref.

[99] 

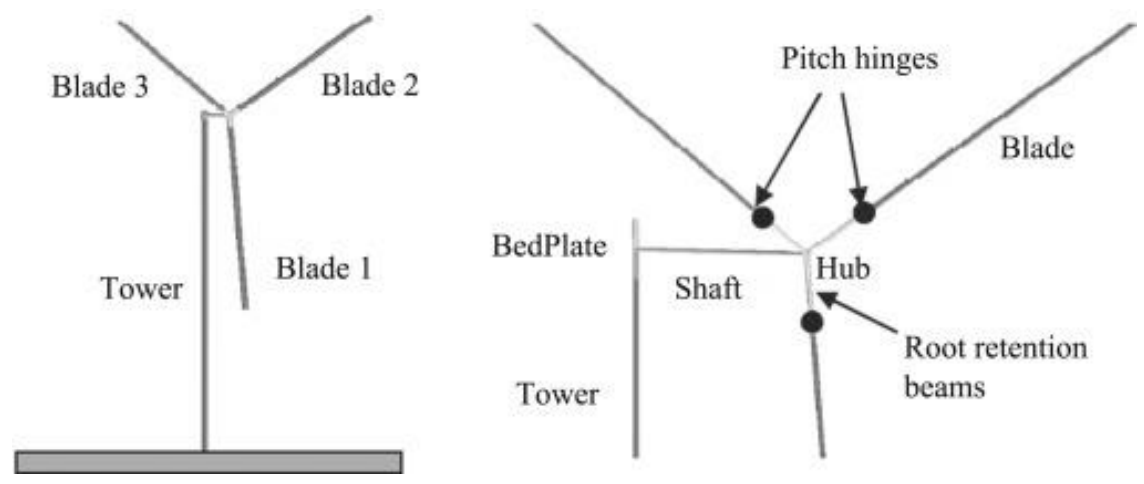

Figure 17. An example of wind turbine configuration based on MBD; reproduced from Ref. [102] 


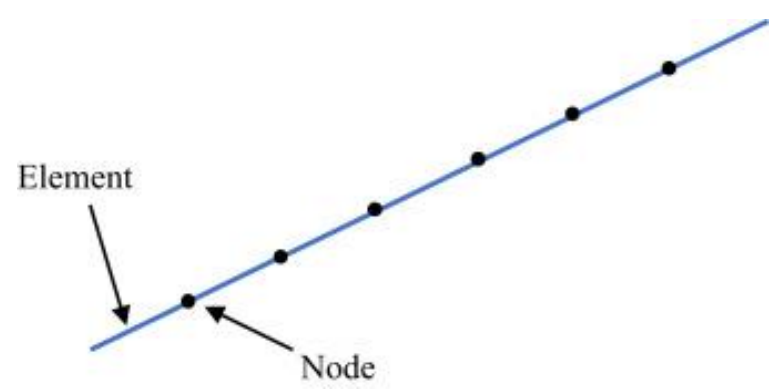

Figure 18. Elements and nodes in 1D FEM 

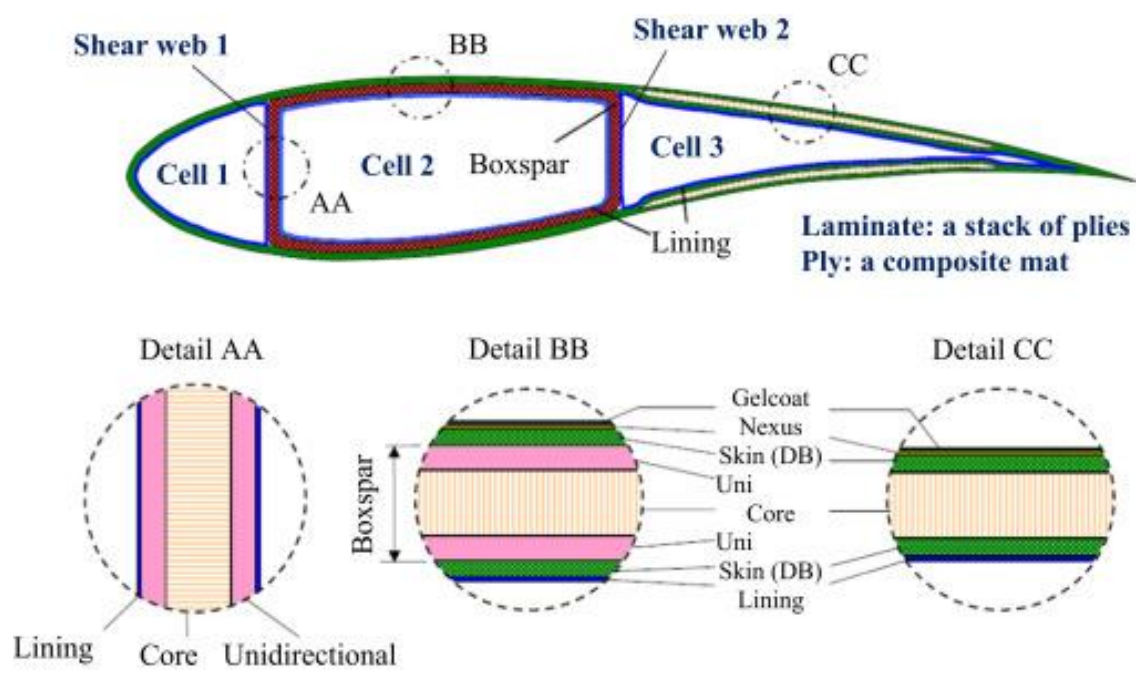

Figure 19. Structural layout of a typical blade cross-section; reproduced from Ref.

[103] 


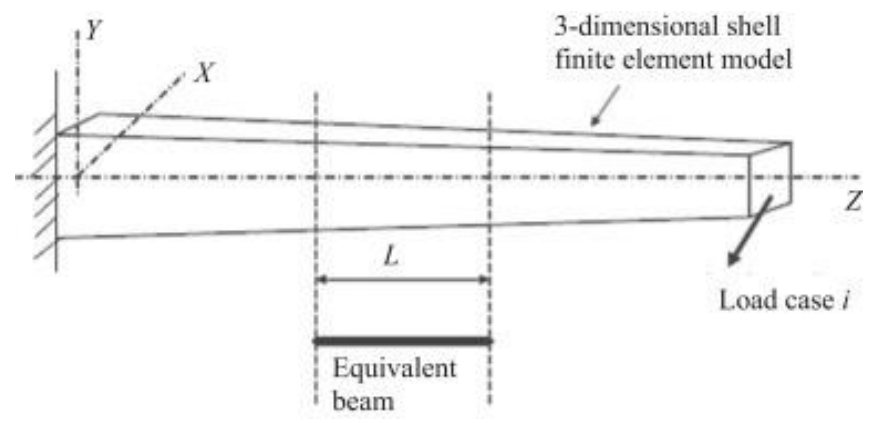

Figure 20. Three-dimensional blade model and equivalent beam element in BPE;

reproduced from Ref. [105] 


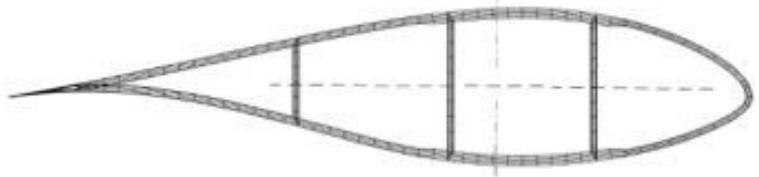

Figure 21. An example of 2D mesh generated by airfoil2BECAS [110] 


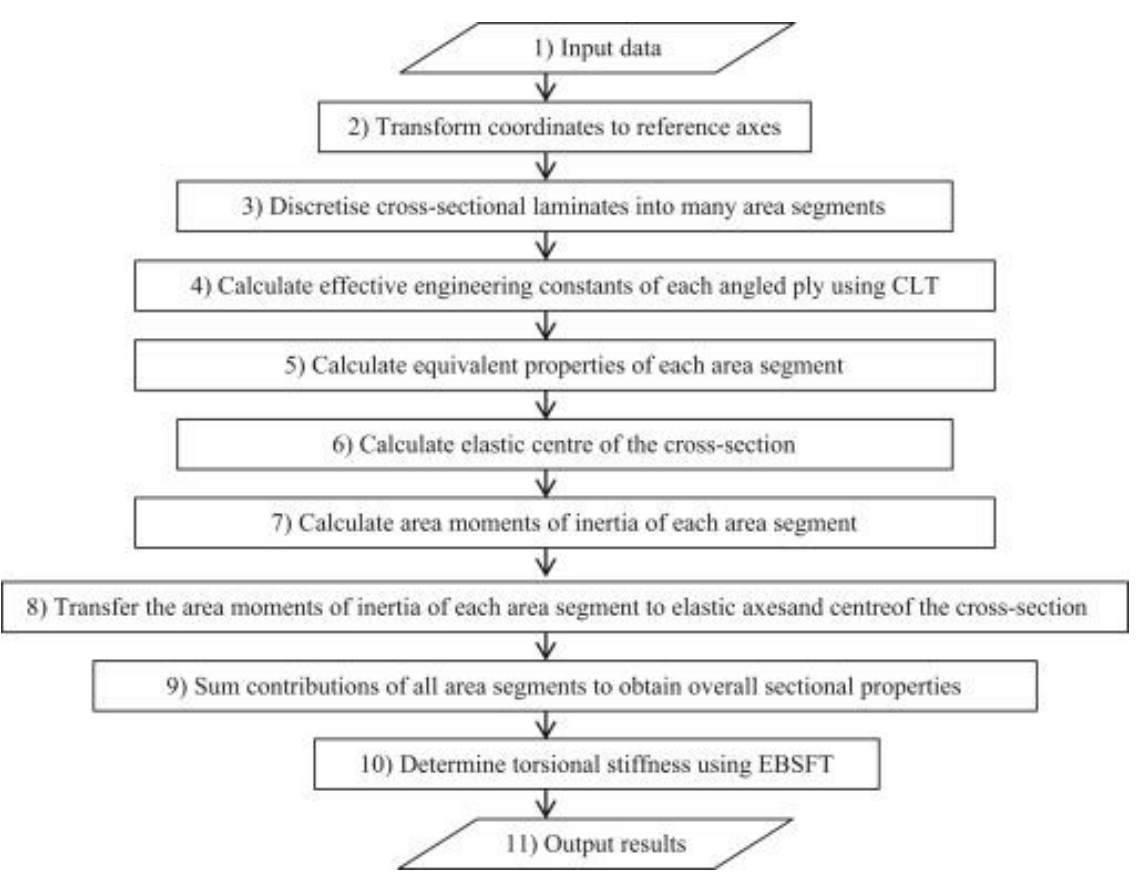

Figure 22. Flowchart of CBCSA [118] 
Table 1. Overview of wind turbine aeroelastic models

\begin{tabular}{lllll}
\hline Name & \multicolumn{2}{c}{$\begin{array}{c}\text { Structural part } \\
\text { Discretisation } \\
\text { Blade } \\
\text { representation }\end{array}$} & $\begin{array}{l}\text { Aerodynamic part } \\
\text { method }\end{array}$ & $\begin{array}{l}\text { Requires blade } \\
\text { cross-sectional } \\
\text { properties as } \\
\text { input? }\end{array}$ \\
\hline ADAMS/WT & 1D beam & MBD & BEM & Yes \\
FAST & 1D beam & Modal approach & BEM & Yes \\
FLEX5 & 1D beam & Modal approach & BEM & Yes \\
GAST & 1D beam & 1D FEM & Free-wake vortex & Yes \\
GH-Bladed & 1D beam & Modal approach & BEM & Yes \\
HAWC2 & 1D beam & MBD & BEM & Yes \\
PHATAS & 1D beam & 1D FEM & BEM & Yes \\
\hline
\end{tabular}


Table 2. Comparison of aerodynamic models

\begin{tabular}{lllll}
\hline & BEM & Vortex & Actuator type & CFD \\
model & model & model & model \\
\hline Accuracy & $* * *$ & $* * *$ & $* * *$ & $* * * *$ \\
Computational speed & $* * * *$ & $* * *$ & $* *$ & $*$ \\
Airfoil aerodynamic data required? & Yes & Yes & Yes & No \\
Viscous effects included? & Yes & No & Yes & Yes \\
\hline
\end{tabular}


Table 3. Comparison of discretisation methods

\begin{tabular}{llll}
\hline & Modal approach & MBD & 1D FEM \\
\hline Accuracy & $*$ & $* *$ & $* * *$ \\
Computational speed & $* * *$ & $* *$ & $*$ \\
Number of DOFs & $*$ & $* *$ & $* * *$ \\
Prescribed modal shapes required? & Yes & No & No \\
\hline
\end{tabular}


Table 4. Comparison of cross-sectional analysis models

\begin{tabular}{llll}
\hline & $\begin{array}{l}\text { 3D FEM-based } \\
\text { model }\end{array}$ & $\begin{array}{l}\text { 2D FEM-based } \\
\text { model }\end{array}$ & $\begin{array}{l}\text { CLT-based } \\
\text { model }\end{array}$ \\
\hline $\begin{array}{l}\text { Accuracy } \\
\text { Computational speed }\end{array}$ & $* * *$ & $* *$ & $*$ \\
$\begin{array}{l}\text { Post-processing of force-displacement data } \\
\text { required? }\end{array}$ & Yes & No & No \\
$\begin{array}{l}\text { Preprocessor (used to generate input files) } \\
\text { required? }\end{array}$ & No & Yes & No \\
\hline
\end{tabular}

